\title{
I,25-Dihydroxyvitamin D Inhibits Osteoarthritis by Modulating Interaction Between Vitamin D Receptor and NLRP3 in Macrophages
}

\author{
Ao Duan (1D ${ }^{1, *}$ \\ Zemeng $\mathrm{Ma}^{2, *}$ \\ Wanshun Liu' ${ }^{\mathrm{l}, *}$ \\ Kai Shen ${ }^{1} *$ \\ Hao Zhou',* \\ Shunbing Wang ${ }^{3}$ \\ Renyi Kong ${ }^{4}$ \\ Yuqi Shao ${ }^{5}$ \\ Yunzi Chen ${ }^{2}$ \\ Wei Guo ${ }^{6}$ \\ Feng Liu'
}

'Department of Orthopedics, The First Affiliated Hospital of Nanjing Medical University, Nanjing, 210029, Jiangsu, People's Republic of China; ${ }^{2}$ Key Laboratory of Immune Microenvironment and Disease, Department of Immunology, Nanjing Medical University, Nanjing, 2 I I 100, People's Republic of China; ${ }^{3}$ Department of Rheumatology and Immunology, Affiliated Hospital of Hospital of North Sichuan Medical College, Nanchong, 637000, People's Republic of China; ${ }^{4}$ Department of Orthopedics, Xincheng Hospital of Traditional Chinese Medicine, Maanshan, 243I3I, Anhui, People's Republic of China; ${ }^{5}$ Department of Orthopedics, The Second Affiliated Hospital of Xuzhou Medical University, Xuzhou, 221006, Jiangsu, People's Republic of China; ${ }^{6}$ Department of Urology, The Affiliated Wuxi No.2 People's Hospital of Nanjing Medical University, Wuxi, 2I 4002, Jiangsu, People's Republic of China

*These authors contributed equally to this work

Correspondence: Wei Guo; Feng Liu Email weiguo_gavin@I63.com; njliuf@I63.com
Background: Osteoarthritis (OA) is the most prevalent chronic joint disease globally. Loss of extracellular matrix (ECM) by chondrocytes is a classic feature of OA. Inflammatory cytokines, such as interleukin-1 $\beta$ (IL-1 $\beta$ ) and interleukin-18 (IL-18), secreted mainly by macrophages, promote expression of matrix degrading proteins and further aggravate progression of OA. 1,25-dihydroxyvitamin D (1,25VD) modulates inflammation thus exerting protective effects on cartilage tissue. However, the underlying mechanisms of $1,25 \mathrm{VD}$ activity have not been fully elucidated.

Methods: The destabilization of the medial meniscus (DMM)-induced mice model of OA was established to investigate the protective effects of $1,25 \mathrm{VD}$ by micro-CT and Safranin-O and Fast Green staining. And the co-culture system between THP-1 cells and primary chondrocytes was constructed to explore the effects of vitamin D receptor (VDR) and $1,25 \mathrm{VD}$ on chondrogenic proliferation, apoptosis, and migration. The immunofluorescence staining and Western blot analysis were used to detect the expressions of ECM proteins and matrix degradation-associated proteases. Enzyme-linked immunosorbent assay (ELISA) was used to examine the expression levels of inflammatory cytokines.

Results: The findings of the study showed that $1,25 \mathrm{VD}$ prevented cartilage degeneration and osteophyte formation by inhibiting secretion of inflammatory cytokines in OA mice model. These protective effects were exerted through the vitamin D receptor (VDR). Further studies showed that $1,25 \mathrm{VD}$ increased ubiquitination level of NLRP3 by binding to VDR, resulting in decrease in IL-1 $\beta$ and IL-18 secretion. These findings indicate that $1,25 \mathrm{VD}$ binds to VDR thus preventing chondrogenic ECM degradation by modulating macrophage NLRP3 activation and secretion of inflammatory cytokines, thus alleviating OA progression.

Conclusion: Here, our study suggests that $1,25 \mathrm{VD}$, targeting to VDR, prevents chondrogenic ECM degradation through regulating macrophage NLRP3 activation and inflammatory cytokines secretion, thereby alleviating OA. These findings provide information on a novel molecular mechanism for application of $1,25 \mathrm{VD}$ as OA therapy.

Keywords: osteoarthritis, 1,25-dihydroxyvitamin D, vitamin D receptor, NLRP3, inflammatory cytokines

\section{Introduction}

Osteoarthritis (OA) is a common joint-related disease globally and it causes motor limitation and disability in the elderly. ${ }^{1}$ Several factors lead to OA, such as age, ${ }^{2}$ joint injury, ${ }^{3}$ hip dysplasia, ${ }^{4,5}$ femoroacetabular impingement morphology, ${ }^{6,7}$ varus (or valgus) knee alignment, ${ }^{8,9}$ and leg length inequality. ${ }^{10}$ Notably, age and joint injury are the most common factors leading to OA. ${ }^{2,3}$ Pathological changes of OA 
include articular cartilage degeneration, osteophyte formation and subchondral bone sclerosis. ${ }^{11,12}$ Chondrocytes are the only cell type residing in cartilage and play a crucial role in articular cartilage composition. The extensive extracellular matrix (ECM) comprises type II collagen alpha 1 (COL2A1) and structural proteoglycan aggrecan, which surrounds the chondrocytes. ${ }^{13}$ Previous studies report that increase of articular cartilage ECM degradation is implicated in OA progression. ${ }^{14,15}$ A disintegrin-like and metalloproteinase domain with thrombospondin-1 motifs 5 (ADAMTS5) is involved in pathogenesis of osteoarthritis as it promotes degradation of aggrecan. In addition, matrix metalloproteinase 13 (MMP13) are major enzymes involved in degradation of the cartilage matrix. ${ }^{16,17}$ Clinical studies and basic studies report that ADAMTS5 and MMP13 exert key effects on articular cartilage degeneration, and their expression is significantly upregulated in OA. ${ }^{18-20}$ Therefore, protection of chondrogenic ECM from degradation is an important strategy in OA treatment.

Several studies report that OA is driven by inflammation-related pro-inflammatory cytokines such as IL-1 $\beta$, and IL-18, which actively remodel the ECM of chondrocytes by significantly upregulating expression of matrix degrading proteins. ${ }^{21,22}$ Moreover, macrophages are the major immune cells that present in synovial lining and exert important functions in maintaining immune homeostasis. ${ }^{23}$ IL-1 $\beta$ and IL-18 secreted by macrophages are mainly produced from macrophage nod-like receptor pyrin domain 3 inflammasome (NLRP3). ${ }^{24,25}$ NLRP3 activation has been widely explored in recent years and is associated with OA synovial inflammation. ${ }^{26,27}$ Upon activation, NLRP3 assembles inflammasome complex and the effector pro-caspase-1, which mediates proteolytic cleavage of pro-caspase- 1 into active caspase- 1 and converts the cytokine precursors pro-IL-1 $\beta$ and pro-IL-18 into biologically active IL- $1 \beta$ and IL-18. ${ }^{27}$ Secretion of IL- $1 \beta$ and IL-18 promotes degradation of ECM. ${ }^{12,28}$ Therefore, inhibition of NLRP3 activation thus reducing inflammatory cytokines release can abrogate ECM degradation.

Previous studies report that $1,25 \mathrm{VD}$, the active form of vitamin $\mathrm{D}$, exerts immunologic activities on multiple components of the innate and adaptive immune system, including inflammation response. ${ }^{29}$ VDR are expressed by most immune cells, including macrophages. Vitamin D and VDR signaling pathway play a suppressive role on inflammatory response, reducing macrophage activation and secretion of inflammatory cytokines. ${ }^{30}$ However, studies have not explored whether vitamin D exerts protective effects on OA progression and the mechanism related to the VDR signaling pathway has not been fully elucidated.

In the current study, destabilization of the medial meniscus (DMM) induced OA model was established to explore the potential protective effects of $1,25 \mathrm{VD}$. The finding showed that $1,25 \mathrm{VD}$, the ligand of VDR, modulated interaction between VDR and NLRP3 in macrophages (MФ), and abrogated secretion of inflammatory cytokines (IL-1 $\beta$ and IL-18), resulting in inhibition of chondrogenic ECM degradation thus ultimately preventing pathological progression of OA.

\section{Materials and Methods}

\section{Experimental Animals and Procedures}

Male C57BL/6 mice (10 weeks) were obtained from the Animal Resource Center of the Faculty of Medicine, Nanjing Medical University for use in the current study. $\mathrm{VDR}^{-/-}$mice were obtained from Yunzi Chen Laboratory. All animal procedures were performed following the guidelines by the Animal Protection and Use Committee of Jiangsu Experimental Animal Association. The study was approved by the Animal Protection and Ethics Committee of Nanjing Medical University (No. IACUC2006052). OA induction was conducted by destabilization of the medial meniscus (DMM) on the right knee. Anterior cruciate ligament and medial meniscus were removed during DMM surgery. All C57 mice aged 8 weeks were randomly divided into 3 groups: Sham group, OA +Vehicle group and OA+Vitamin D group, with 13 mice in each group. Sham group only underwent sham operation, that is, the skin and muscle inside the right knee joint were cut with a surgical blade, and then sutured. The mice in $\mathrm{OA}+$ Vehicle group and $\mathrm{OA}+$ Vitamin $\mathrm{D}$ group received DMM operation and were fed PBS or Vitamin D $(0.25 \mathrm{ug} /$ $\mathrm{kg} / \mathrm{d}$ ) for six weeks. The grouping and treatment of $\mathrm{VDR}^{-1}$ mice were the same as C57 mice. Each mouse was treated with buprenorphine $(0.05 \mathrm{mg} / \mathrm{kg})$ to relieve pain and gentamicin $(5 \mathrm{mg} / \mathrm{kg})$ to prevent infection after the operation. Three days after DMM operation, all animals were forced to jog on a specific treadmill at $10 \mathrm{~m} / \mathrm{min}$ for 40 min per day for 6 weeks.

\section{Administration of Drugs}

1,25VD and vitamin D were purchased from SigmaAldrich (17943 and C2150000, respectively). Phorbol myristate acetate (PMA), LPS And Nigericin (Nig) were 
purchased from Sigma-Aldrich (P1585, 916374 and N7143, respectively). Vitamin D was dissolved in PBS buffer and $0.25 \mathrm{ug} / \mathrm{kg} / \mathrm{d}$ administered to mice by intragastrical administration as previously reported. ${ }^{31}$ In addition, $1,25 \mathrm{VD}$ was dissolved in absolute ethyl alcohol, to achieve different concentrations of 1,25VD at $4 \mu \mathrm{M}, 20 \mu \mathrm{M}$ and $100 \mu \mathrm{M}$ which were used to treat cells for $48 \mathrm{~h}$. PMA was dissolved in dimethyl sulfoxide to achieve a concentration of $1 \mu \mathrm{M}$ which was used to treat cells. LPS was dissolved in PBS buffer and was used to treat cells at a concentration of $200 \mathrm{ng} / \mathrm{mL}$. Nig was dissolved in absolute ethyl alcohol and was used to treat cells at a concentration of $10 \mu \mathrm{M}$. Activating NLRP3 in THP-1 usually requires two signals, one is LPS and the other is Nigericin. ${ }^{32}$ LPS activates inflammatory factor precursors and NLRP3 through Nf-kb. ${ }^{32,33}$

\section{Cell Culture and Treatment of Cells}

Isolation and incubation of human chondrocytes was conducted as described previously. ${ }^{34}$ Knee cartilages were obtained from clinical cases who underwent total knee arthroplasty (TKA). The study protocol was approved by the research ethics committee of the First Affiliated Hospital of Nanjing Medical University (No. 2021-SR -027). All OA patients without additional systemic disorders provided informed consent. Cartilage tissue was washed three times with PBS buffer supplemented with $1 \%$ penicillin-streptomycin $(\mathrm{P} / \mathrm{S})(\mathrm{Gibco})$. Cartilage tissues were then cut using a surgical blade into cubes with a rib length of $1 \mathrm{~mm}$. Cartilage tissues were digested with PBS containing 2\% trypsin (Gibco) for $30 \mathrm{~min}$, then with complete medium containing $2 \mathrm{mg} / \mathrm{mL}$ collagenase type II (Gibco) for 12-16 h. The digestion was terminated and chondrocytes were filtered through a cell sieve and centrifuged 3 times (1000 rpm, $5 \mathrm{~min}$ ) to obtain primary chondrocytes. Chondrocytes were cultured in Dulbecco's Modified Eagle Medium/Nutrient Mixture F-12 (DMEM/ F12, Gibco) supplemented with $10 \%$ fetal bovine serum (FBS) (Gibco) and 1\% penicillin/streptomycin (Gibco). Cells were then incubated at a constant temperature of $37{ }^{\circ} \mathrm{C}$ and $5 \% \mathrm{CO} 2$ for subsequent experiments. All of the above procedures were performed under aseptic conditions.

THP-1, a promonocytic cell line, was obtained from American Type Culture Collection (Nr. TIB-202). THP-1 cells were cultured in RPMI 1640 medium (Gibco) supplemented with $10 \%$ FBS and $1 \%$ penicillin/streptomycin.

\section{Cell Transfection}

THP-1 cells were used for cell transfection experiment after adherence. Adherence of THP-1 was induced by PMA. Adherent THP-1 cells were transfected with siRNAVDR (GenePharma, China) and Lipofectamine 2000 (Invitrogen, United States). ${ }^{35}$ The siRNA-VDR sequence used was CCTCCAGTTCGTGTGAATGAT; ATCATTCA CACGAACTGGAGG. The siRNA-NLRP3 sequence used was GCTCATATCATCATTCCCGCT; ACCAGCTACAA AAAGCATGGA.

\section{Micro-Computed Tomography (Micro-CT) Joint Imaging}

Knee joint tissues of mice were imaged using Skyscan 1176 Micro-CT equipment (Bruker, Billerica, MA, USA). 3D images were reconstructed using SkyScan volumetric NRecon reconstruction software version 1.6 (Bruker, Billerica, MA, USA). The region of interest (ROI) of subchondral bone was selected after 3D reconstruction. Osteophyte score was analyzed by two experimenters who were blinded to the treatments as described previously. ${ }^{36}$

\section{Immunofluorescence Staining}

Tissue sections were processed as previously described. ${ }^{37}$ Mice tissue samples were perfused with PBS and 4\% paraformaldehyde until they were stiff. Mouse knee samples were decalcified, dehydrated, and waxed before cutting them into $5 \mu \mathrm{m}$-thick sections for the subsequent experiments. After dewaxing and hydration, tissue slices were blocked in $10 \%$ goat serum/PBST for $1 \mathrm{~h}$ at room temperature and were further incubated with primary antibodies overnight at $4{ }^{\circ} \mathrm{C}$. Primary antibodies used in the experiments included anti-COL2A1 (1: 200, Abcam, ab34712), anti-aggrecan (1:200, Proteintech, 138801-AP), anti-ADAMTS5 (1:200; Abcam, ab182795) and anti-MMP13 (1:200, Proteintech, 18165-1-AP). Samples were incubated with Alexa Fluor 488 or 555 donkey antirabbit secondary antibodies for $1 \mathrm{~h}$ at room temperature. Images were captured with fluorescence microscope (Olympus, Japan) and quantification of protein levels was performed using ImageJ software.

\section{Safranin-O and Fast Green Staining}

Tissue sections were obtained following the protocol described in immunofluorescence staining section. Safranin-O and Fast Green staining was performed using 
Safranin-O and Fast Green FCF Stain Kit (Solarbio) following the manufacturer's instructions. ${ }^{38}$ Images were obtained using a microscope (Olympus, Japan).

\section{Enzyme-Linked Immunosorbent Assay (ELISA)}

ELISA analysis of mice synovial fluid was conducted to explore the expression levels of inflammatory factors in vivo, as previously reported. ${ }^{39}$ Mice joint tissue was homogenized in RIPA buffer and centrifuged at $12,000 \mathrm{rpm}$ for $30 \mathrm{~min}$. The supernatant (SN) was collected for use in ELISA analysis. IL-1 $\beta$, IL-18 and TNF- $\alpha$ levels were determined using ELISA kits (Cloud-Clone Corp, SEA563Hu, SEA064Hu, SEA133Hu).

The cell culture medium in each group and the concentrations of cytokines were determined by ELISA to explore changes in expression levels of inflammatory factors in the SN. IL-1 $\beta$, IL-18 and TNF- $\alpha$ levels were determined using ELISA kits (Cloud-Clone Corp, SCA563Mu, SEA064Mu, SEA133Hu).

\section{Immunoprecipitation Analysis and Immunoblotting}

Immunoprecipitation analysis and immunoblotting were performed following methods described previously. ${ }^{40}$ Cells were lysed in SDS lysis buffer to obtain the wholecell lysates. For co-immunoprecipitation, cells were treated with $500 \mu \mathrm{L}$ RIPA Lysis Buffer followed by incubation at $4{ }^{\circ} \mathrm{C}$ for $30 \mathrm{~min}$. Cells were then centrifuged at $12,000 \times \mathrm{g}$ and $4{ }^{\circ} \mathrm{C}$ for $15 \mathrm{~min}$ and the supernatants were incubated with the appropriate antibodies overnight. Protein A/G-Agarose slurry was added to samples for capturing the immunocomplexes at $4{ }^{\circ} \mathrm{C}$ for $90 \mathrm{~min}$. Samples were centrifuged for $3 \mathrm{~min}$ at $100 \mathrm{rpm}$ with agarose beads. The beads were washed three times with $800 \mu \mathrm{L}$ of ice-cold RIPA buffer and resuspended in $50 \mu \mathrm{L}$ SDS loading buffer. Samples were analyzed by immunoblotting method. Immunoreactivity bands were visualized by ECL reagent and Tanon 5200 Chemiluminescence image analysis system. For the NLRP3 ubiquitination assay, $300 \mu \mathrm{L}$ RIPA buffer was added to cells. Cells were then centrifuged for $10 \mathrm{~min}$. The SN was treated with $1 \%(\mathrm{w} / \mathrm{v}) \mathrm{SDS}$, heated to $100{ }^{\circ} \mathrm{C}$ for $5 \mathrm{~min}$ to dissociate NLRP3 from any associated proteins, and then diluted 10-fold with RIPA buffer before immunoprecipitation.

\section{Proliferation Assay of Chondrocytes}

Chondrocytes were seeded into 24-well plates and cultured to achieve $70-80 \%$ confluence for use in proliferation assay. THP- 1 cells $\left(1.6 \times 10^{4}\right.$ cells $\left./ \mathrm{cm}^{2}\right)$ were seeded in the upper layer of a 24-well transwell chamber (pore size: $0.4 \mu \mathrm{m}$, Corning) whereas PBS buffer was added to the lower chamber. Adherence of THP-1 was induced by PMA. THP-1 cells were then transfected with siRNA-NC or siRNA-VDR. After transfection, cells were treated with complete culture medium containing LPS for $4 \mathrm{~h}$ and then treated with complete culture medium containing LPS \& Nig for $1 \mathrm{~h}$. Cell proliferation was analyzed using EdU kit. ${ }^{41}$ We transferred the transwell chamber into the chondrocytes-adhered 24-well plates to establish the co-culture system and the medium containing the LPS, Nig and EdU $(10 \mu \mathrm{M})$ was replaced; cells were then incubated for 24 h. After incubation, BeyoClick ${ }^{\mathrm{TM} E d U-594}$ Cell Proliferation Detection Kit (Beyotime) was used to determine proliferation rate following the manufacturer's instructions. Cell proliferation of each group was analyzed by fluorescence microscope and flow cytometry.

\section{Apoptosis Analysis of Chondrocytes}

A co-culture system was established following the protocol described in the proliferation assay section (Figure 4). Cells were incubated for $24 \mathrm{~h}$ and analysis was performed using the TUNEL Bright Green Apoptosis Detection Kit (Vazyme) following the manufacturer's instructions. Apoptosis rate of each group was determined using fluorescence microscope and images were analyzed under a fluorescence microscope. Flow cytometry analysis was performed using Annexin V-FITC/PI Apoptosis Detection Kit (Vazyme) following the manufacturer's instructions.

\section{Migration Analysis of Chondrocytes}

THP-1 cells were seeded into 24-well culture plates at a density of $1.6 \times 10^{4}$ cells $/ \mathrm{cm}^{2}$. THP-1 adhesion was induced by treatment with PMA (P1585, sigma-Aldrich). After adherence, THP-1 cells were transfected with siRNA-NC or siRNA-VDR then treated with complete culture medium containing LPS for $4 \mathrm{~h}$ and further treated with complete culture medium containing LPS \& Nig for 1h. Chondrocytes were inoculated in the upper 24-well transwell chamber (pore size: $8 \mu \mathrm{m}$, Corning) at a density of $2 \times 10^{4}$ cells per well. The THP-1 adhered 24well plates were then used to establish a co-culture system, and the medium containing LPS \& Nig was replaced and 
cells incubated for $24 \mathrm{~h}$. After incubation, cells were fixed with $4 \%$ paraformaldehyde for $15 \mathrm{~min}$, stained with $0.5 \%$ crystal violet for $30 \mathrm{~min}$, and washed thrice with PBS. The upper surface of the upper chamber was swabbed to remove cells that had not moved to the surface of the lower chamber. The chondrocytes migration rate of each group was observed under a microscope, and four fields were randomly picked for analysis.

In the scratch assay, the co-culture system was established following the protocol described in the proliferation assay section. Cells were incubated for $48 \mathrm{~h}$ to exclude the effect of cell proliferation on the results. Chondrocytes were treated with mitomycin $(1 \mu \mathrm{g} / \mathrm{mL})$ for $1 \mathrm{~h}$ prior to the experiment. After incubation for $24 \mathrm{~h}$, a sterile pipette tip $(200 \mu \mathrm{L})$ was used to make a scratch on the cell layer. Cells were washed three times with PBS and images were captured at $0 \mathrm{~h}, 24 \mathrm{~h}$, and $48 \mathrm{~h}$ with a microscope.

\section{CCK-8 Assay}

Cell survival rates were estimated using the CCK- 8 kit (keyGEN biotech, Jiangsu, China). Approximately $10^{4}$ cells were seeded in 96-well plates with $100 \mu \mathrm{L}$ medium each well. After $48 \mathrm{~h}$ cultivation, different doses of $1,25 \mathrm{VD}$ was added, respectively. Each well was incubated with $10 \mu \mathrm{g}$ CCK-8 solution for $2 \mathrm{~h}$ away from light before measuring the absorbance at $450 \mathrm{~nm}$ by PerkinElmer's EnSpire Multilabel Plate Reader.

\section{Western Blot Analysis}

Western blot analysis was conducted following a method described previously. ${ }^{42}$ The co-culture system was established in 6-well culture plates following the protocol described in the proliferation assay section. Protein lysates were prepared using RIPA lysis buffer (Beyotime Biotechnology, China). Concentration of protein lysates was quantified using BCA Protein Assay kit (Beyotime Biotechnology, China). Protein samples $(30 \mu \mathrm{g})$ were separated by SDS-PAGE electrophoresis and transferred to PVDF membranes (Roche, Mannheim, Germany). PVDF membranes were blocked with $5 \% \mathrm{BSA} / \mathrm{TBST}$ at room temperature and incubated with primary antibodies overnight at $4{ }^{\circ} \mathrm{C}$. Membranes were washed thrice with TBST then incubated with secondary antibodies at room temperature. Protein bands were visualized using ECL reagent and Tanon 5200 Chemiluminescence image analysis system. The gray value was analyzed by ImageJ. Primary antibodies used in this experiment included anti-COL2A1 (1:2000, Abcam, ab34712), anti-aggrecan (1:2000,
Proteintech, 13880-1-AP), anti-ADAMTS5 (1:2000, Abcam, ab182795), anti-MMP13 (1:2000, Proteintech, 18165-1-AP), anti-NLRP3 (1:1000, Adipogen, AG-20B0014), anti-IL-1 $\beta$ (1:1000, R\&D Systems, AF-401-NA), anti-Ubiquitin (1:1000, Santa Cruz, sc-8017), anti-VDR (1:1000, Santa Cruz, sc-13133), anti- $\beta$-actin (1:1000, Santa Cruz, sc-47778), anti-ASC (1:1000, Santa Cruz, sc-514414) and anti-Caspase-1 (1:1000, Abcam, ab108362).

\section{Statistical Analysis}

All experimental data were expressed as mean \pm SD. GraphPad Prism 8.02 software (GraphPad Software, Inc., San Diego, CA) was used for statistical analysis. Statistical analyses were performed using one-way ANOVA with post hoc Tukey's test for multiple comparisons. Differences were regarded as statistically significant when $\mathrm{p}$-value was $<0.05$.

\section{Results}

\section{Vitamin D Alleviates Knee Osteoarthritis in DMM-Induced OA Mice Model}

A DMM-induced OA mice model was established to explore the protective effects of vitamin D on OA. Knee sections of mice from each group were stained with Safranin-O and Fast Green to explore structural changes of articular cartilage, subchondral bone and bone tissue. The findings showed that treatment with vitamin D ameliorated the rough cartilage surface and the severely exposed subchondral bone compared with the vehicle group (Figure 1A), indicating the protective effect of vitamin D. In addition, vitamin D treatment significantly reduced the Osteoarthritis Research Society International (OARSI) score for mice in the vitamin D group compared with the vehicle group (Figure 1B). Moreover, micro-CT was performed to explore formation of osteophyte in each group. Micro-CT results showed that osteophyte volume was significantly higher in the vehicle group compared with the sham group. However, treatment with vitamin $\mathrm{D}$ abrogated the increase in osteophyte formation in OA model mice (Figure 1A). Osteophyte score analysis showed that the osteophyte score of the vitamin D group was significantly lower than that of the vehicle group. Findings from osteophyte score analysis were consistent with the micro-CT analysis (Figure 1C). These findings indicate that vitamin $\mathrm{D}$ inhibits osteophyte production in DMM-induced OA mice model. 
A

Safranin-O \& Fast Green
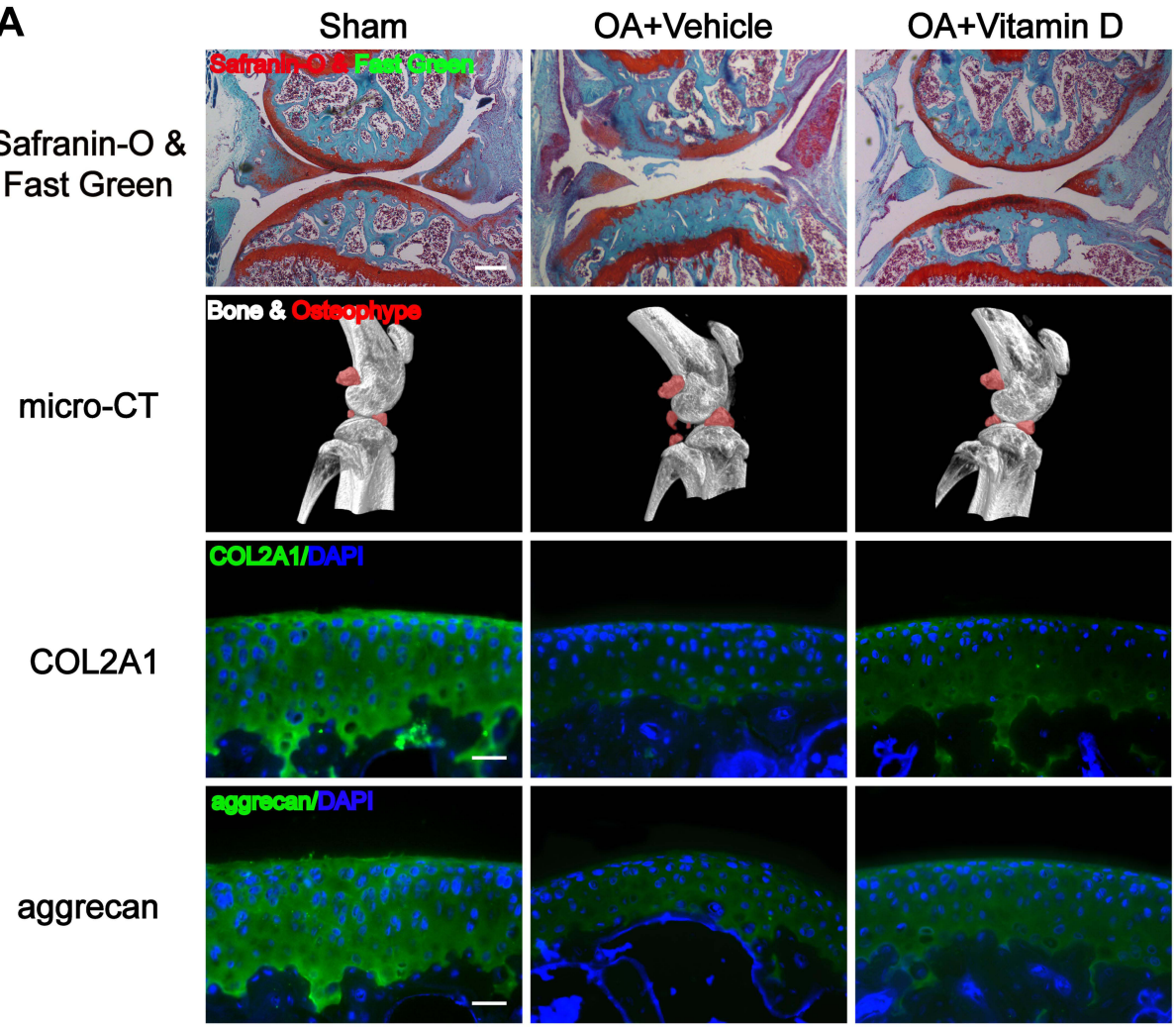

B
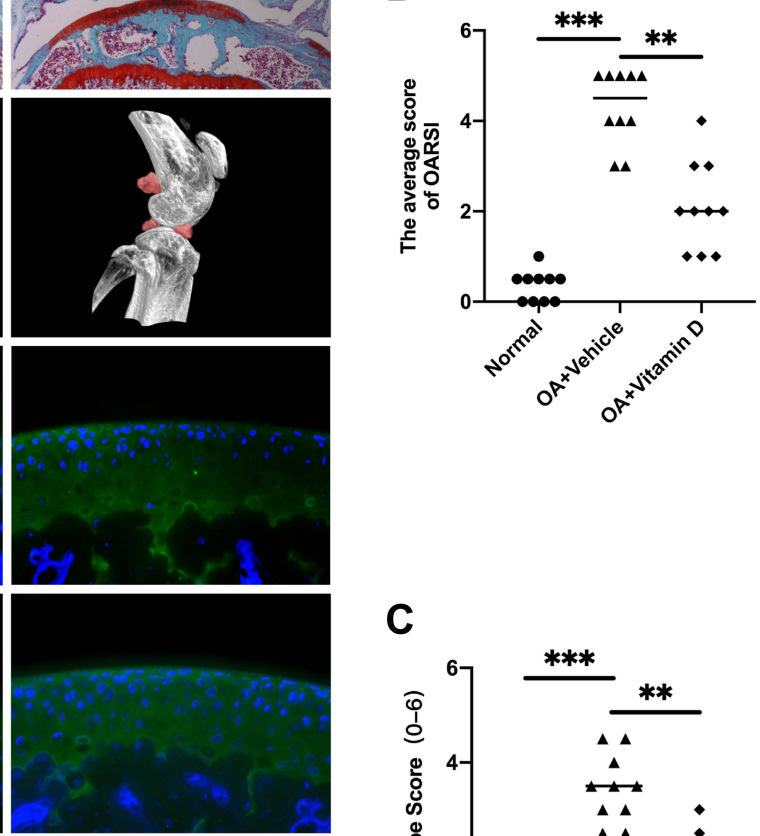

C

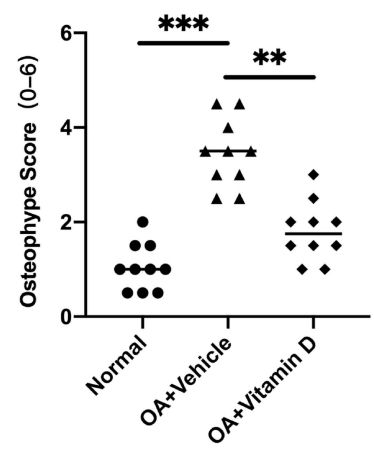

ADAMTS5
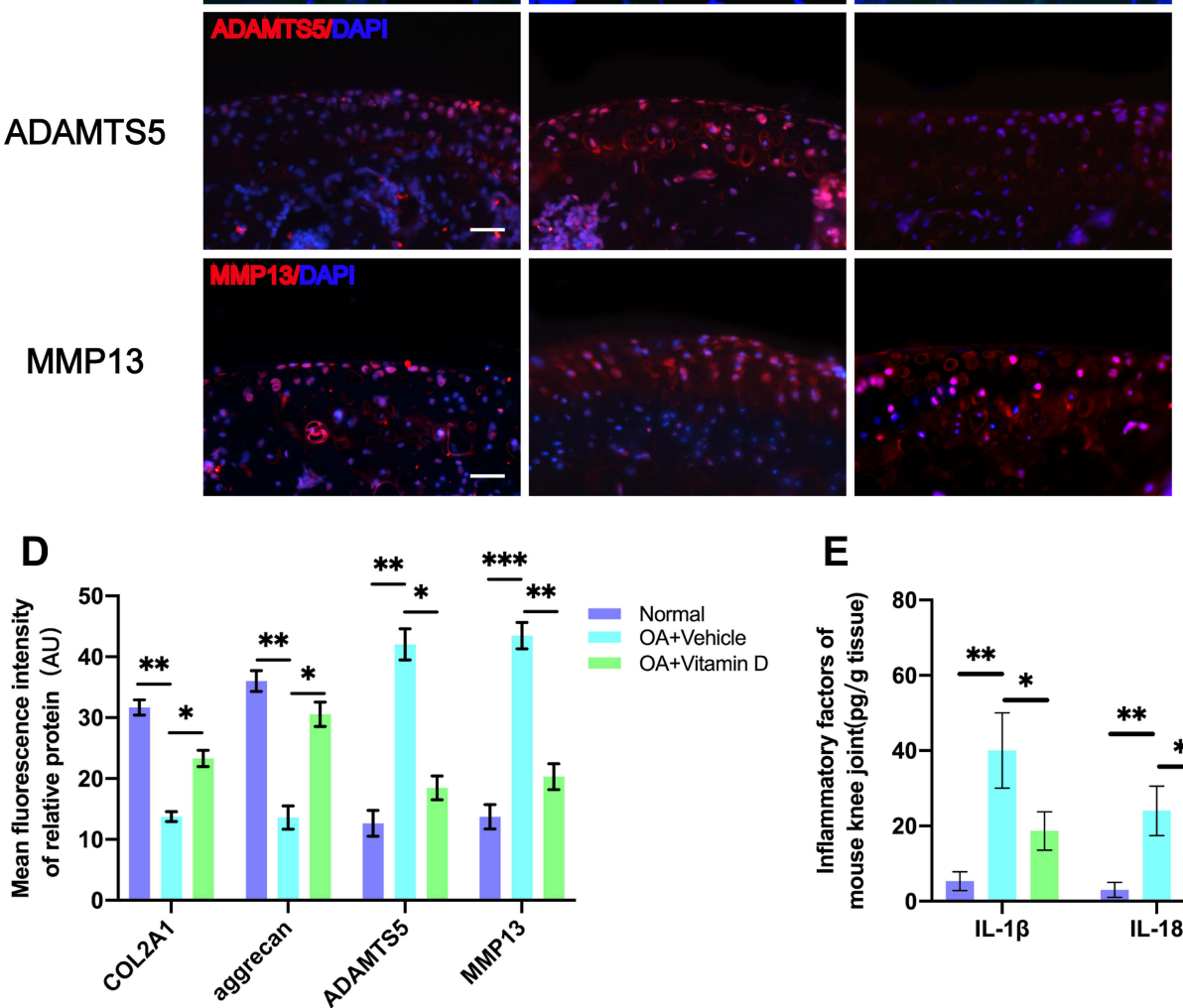

E

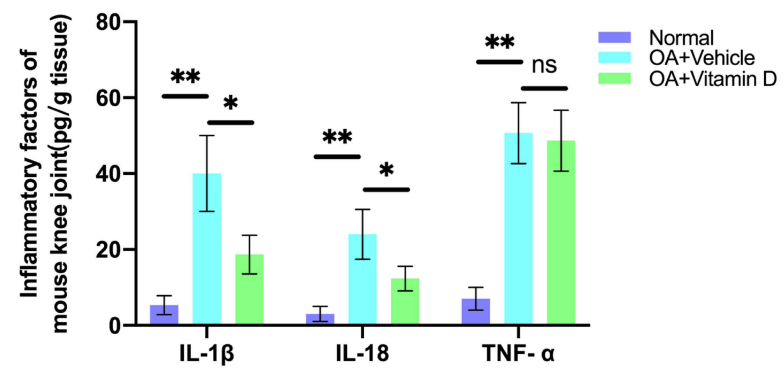

Figure I Vitamin D alleviates knee osteoarthritis in DMM-induced OA mice model. (A) Severity of DMM-induced OA mice model as determined by Safranin-O and Fast Green, and micro-CT analysis. Expression levels of COL2AI, aggrecan, MMPI3 and ADAMTS5 as determined by immunofluorescence staining. Scale bar = $400 \mu \mathrm{m}$ for Safranin-O \& Fast Green staining; Scale bar $=100 \mu \mathrm{m}$ for immunofluorescence staining. (B) OARIS scores in each group ( $\mathrm{n}=10$, one-way ANOVA). (C) Osteophyte score of each group ( $n=10$, one-way ANOVA). (D) Quantification of mean fluorescence intensity of COL2AI, aggrecan, MMPI 3 and ADAMTS5 in each group ( $n=10$, one-way ANOVA). (E) Levels of IL-I $\beta$, IL-I 8 and TNF- $\alpha$ in mouse knee tissue as determined by ELISA ( $n=3$, one-way ANOVA). Data are presented as mean \pm SD. ns, no significance; $*_{\mathrm{p}}<0.05 ; *_{\mathrm{p}}<0.01$; ***p $<0.001$. 
Degradation of ECM protein is an important pathological characteristic of OA. ${ }^{43}$ Therefore, immunofluorescence analysis was performed to explore expression levels of ECM proteins (COL2A1 and aggrecan) and ECM degradation-associated proteases (MMP13 and ADAMTS5). Immunofluorescence results showed that the expression levels of COL2A1 and aggrecan of the vehicle group were significantly lower than those of the sham group. Meanwhile, the expressions of MMP13 and ADAMTS5 were markedly elevated in the vehicle group, which did not display in the sham group. Notably, treatment with vitamin D significantly alleviated the decreased expression of ECM proteins (COL2A1 and aggrecan), and abrogated the elevated expression of ECM degradationassociated proteases (MMP13 and ADAMTS5) (Figure 1A and D).

OA is characterized by inflammatory response, ${ }^{44}$ thus inflammatory factors may be responsible for the phenomena observed. Therefore, ELISA kits were used to further explore the levels of major inflammatory factors in each group. ELISA results showed that the vitamin D treatment group significantly reduced the increased levels of IL-1 $\beta$ and IL-18 after DMM surgery compared with the levels of the vehicle group. However, although the secretion of TNF- $\alpha$ was significantly elevated in OA model group, the level was not significantly changed after vitamin $\mathrm{D}$ treatment (Figure 1E). These findings indicate that vitamin D effectively abrogates development of $\mathrm{OA}$ in the DMM-induced OA mice model.

\section{Vitamin D Exerts Joint Protective Effects Through VDR}

VDR knockout mice (VDR ${ }^{-/-}$mice) were used to explore whether vitamin $\mathrm{D}$ exerted its protective effects through VDR. The findings showed that vitamin $\mathrm{D}$ treatment did not protect cartilage against degeneration in $\mathrm{VDR}^{-/}$mice that have undergone DMM surgery (Figure 2A). OARSI scores showed that vitamin $\mathrm{D}$ did not exert protective effect on DMM-induced OA VDR ${ }^{-/}$mice model (Figure 2B). In addition, formation of osteophytes was evaluated by micro-CT. The findings indicated that vitamin D did not exhibit the inhibitory effect on osteophyte formation in DMM-induced $\mathrm{OA} \mathrm{VDR}^{-/}$mice model (Figure 2A). Findings from osteophyte score analysis were consistent with the micro-CT analysis (Figure 2C).

Further, the expression levels of COL2A1 and aggrecan were explored to determine the status of ECM degradation. Notably, expression levels of COL2A1 and aggrecan were not significantly increased after treatment with vitamin $\mathrm{D}$ in the DMM-induced $\mathrm{OA} \mathrm{VDR}^{-/}$mice compared with the levels in the vehicle group (Figure 2A and D). ECM degradation-associated proteases (MMP13 and ADAMTS5) were not significantly downregulated after vitamin D treatment in the DMM-induced OA $\mathrm{VDR}^{-/-}$mice model compared with the vehicle group (Figure 2A and D).

Expression levels of inflammatory cytokines in each group were explored using ELISA kits. Expression of IL$1 \beta$ and IL-18 was not significantly downregulated in DMM-induced OA VDR ${ }^{-/-}$mice after treatment with vitamin D compared with the levels in DMM-induced OA mice (Figure 2E). The result was different from the results observed in DMM-induced OA mice treated with vitamin D. These findings indicate that vitamin D exerts antarthritic effects in a VDR dependent manner.

\section{VDR Directly Interacts with NLRP3 to Increase Ubiquitination Level of NLRP3}

The findings showed increased IL-1 $\beta$ and IL-18 levels after DMM surgery in mice, indicating activation of NLRP3 inflammasomes. Inflammasome signaling molecule NLRP3 is implicated in progression of OA and is a potential novel biomarker for OA. ${ }^{45}$ Previously reported liquid chromatography-mass spectrometry analysis showed that VDR interacting with NLRP3 VDR can affect NLRP3 in bone marrow-derived macrophage (BMDM). ${ }^{46}$ In addition, co-immunoprecipitation was performed to further confirm the interaction between NLRP3 and VDR in THP-1 cells. The findings showed interaction of VDR and NLRP3 in the LPS group and LPS \& Nig group (Figure 3A). Moreover, consistent findings were obtained through immunofluorescence colocalization of samples from synovial sections of OA patients and normal subjects (Figure 3B).

NLRP3 activates caspase-1 through formation of inflammasome complex, subsequently leading to secretion of pro-inflammatory cytokines. To explore the effects of VDR-NLRP3 interaction on NLRP3 downstream signals, VDR was knocked down in THP-1 cells. The findings showed that VDR knockdown significantly upregulated expression of caspase-1 and IL-1 $\beta$ secretion in SN after LPS \& Nig induced NLRP3 activation (Figure 3C). When both VDR and NLRP3 were knocked down, the levels of IL-1 $\beta$ and caspase-1 decreased significantly compared 


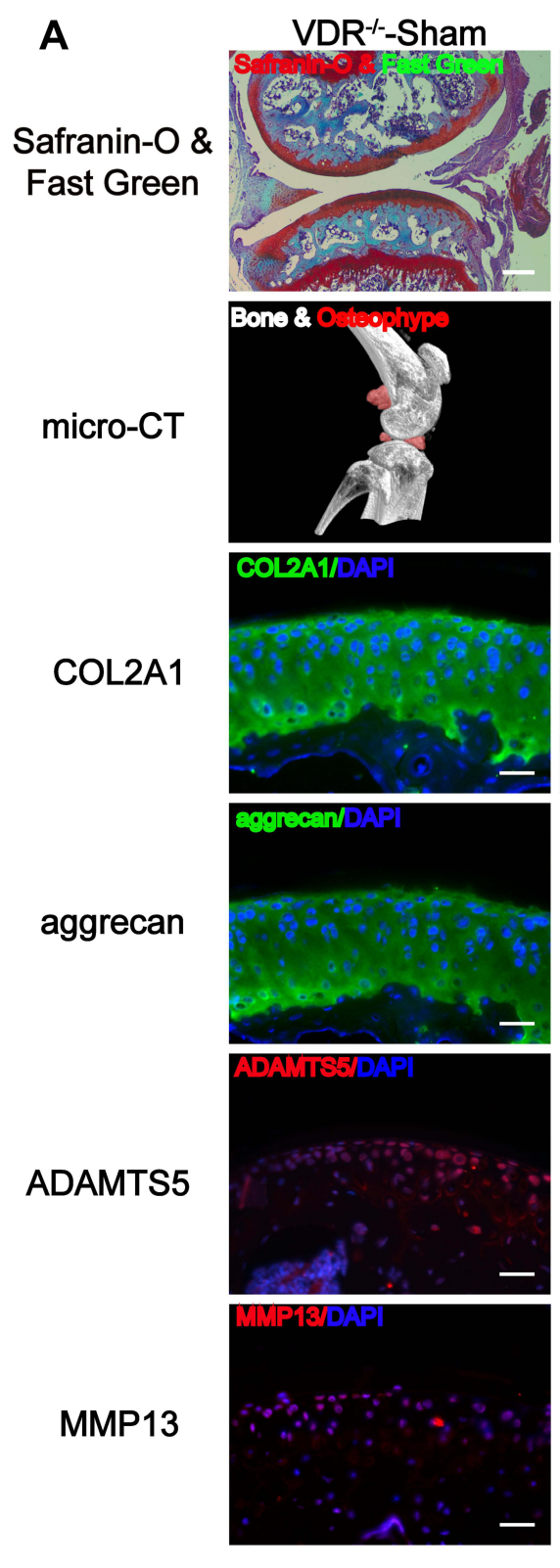

D

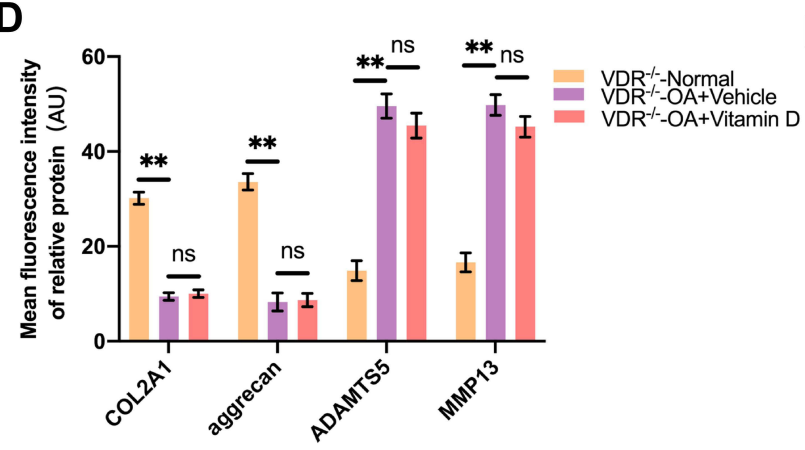

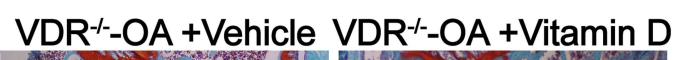
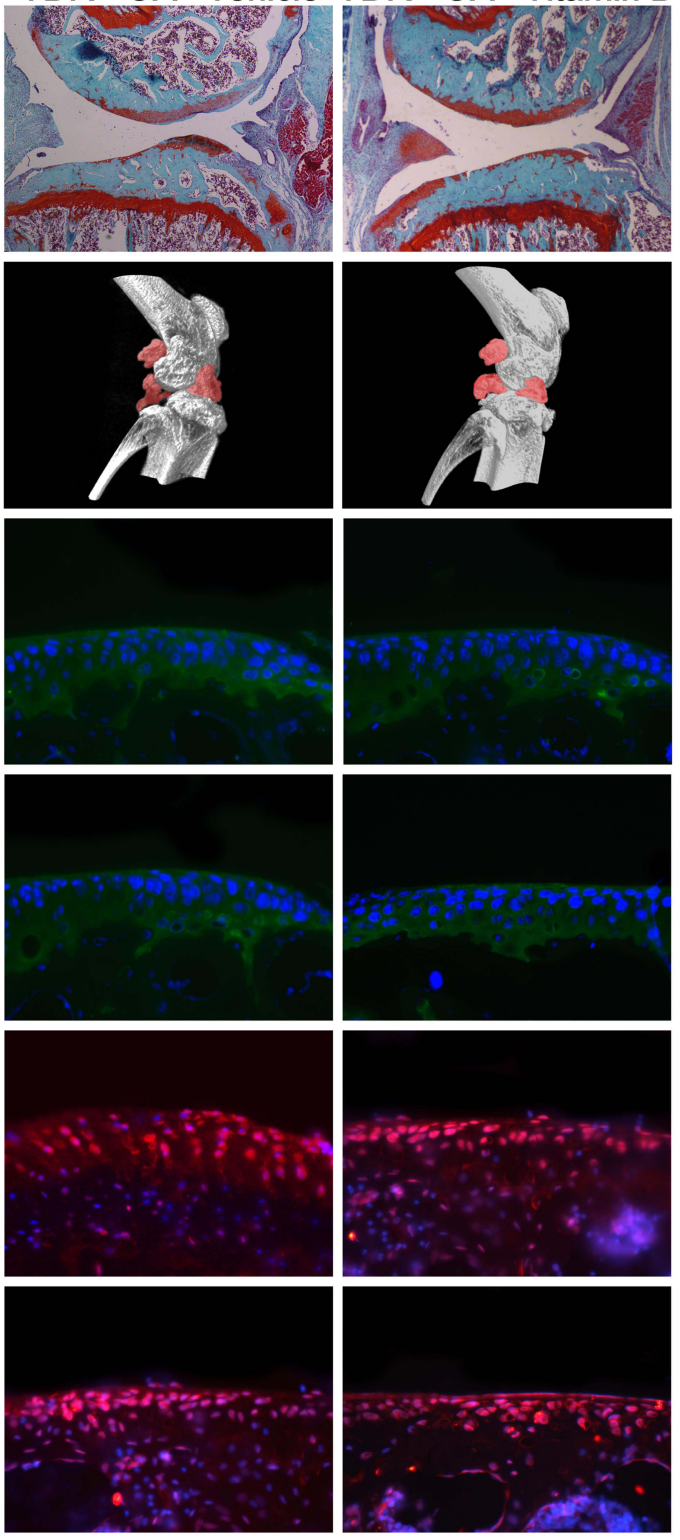

E

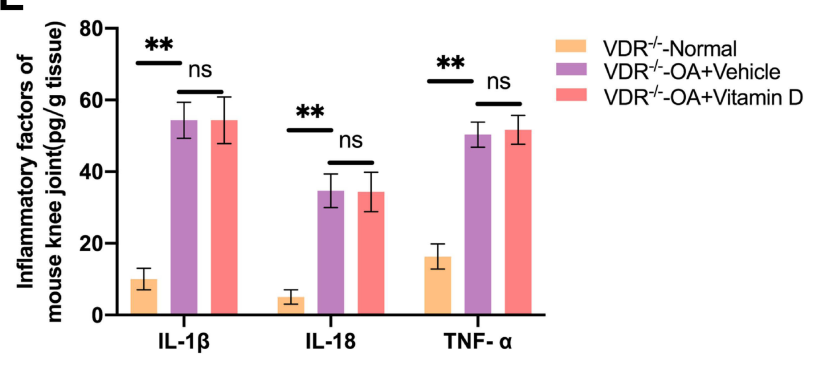

Figure 2 Vitamin D does not exert joint protective effect in DMM-induced OA VDR ${ }^{-1-}$ mice model. (A) Severity of DMM-induced OA VDR ${ }^{-1-}$ mice model as determined by Safranin-O and Fast Green, and micro-CT assays. Expression levels of COL2AI, aggrecan, MMPI 3 and ADAMTS5 as determined by immunofluorescence staining. Scale bar = $400 \mu \mathrm{m}$ for Safranin-O and Fast Green staining; Scale bar $=100 \mu \mathrm{m}$ for immunofluorescence staining. (B) OARIS scores in each group $(\mathrm{n}=10$, one-way ANOVA). (C) Osteophyte score in each group ( $n=10$, one-way ANOVA). (D) Quantification of mean fluorescence intensity of COL2AI, aggrecan, MMPI 3 and ADAMTS5 in each group $(n=10$, one-way ANOVA). (E) ELISA analysis of IL-I $\beta$, IL-I 8 and TNF- $\alpha$ levels in mice knee synovial fluid $(n=3$, one-way ANOVA). Data are presented as mean \pm SD. ns, no significance; $*_{p}^{*}<0.01$. 
A

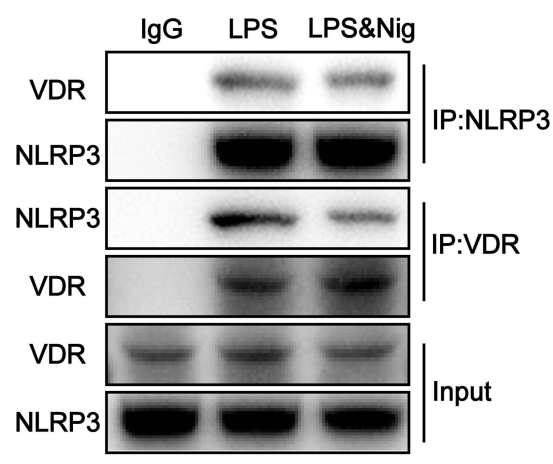

C

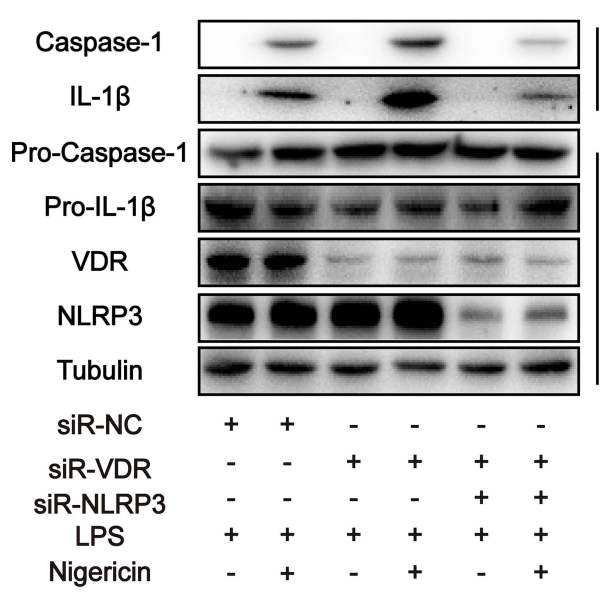

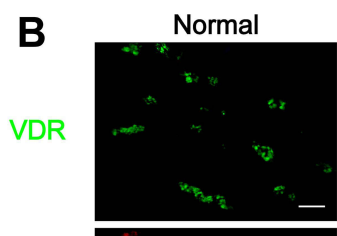
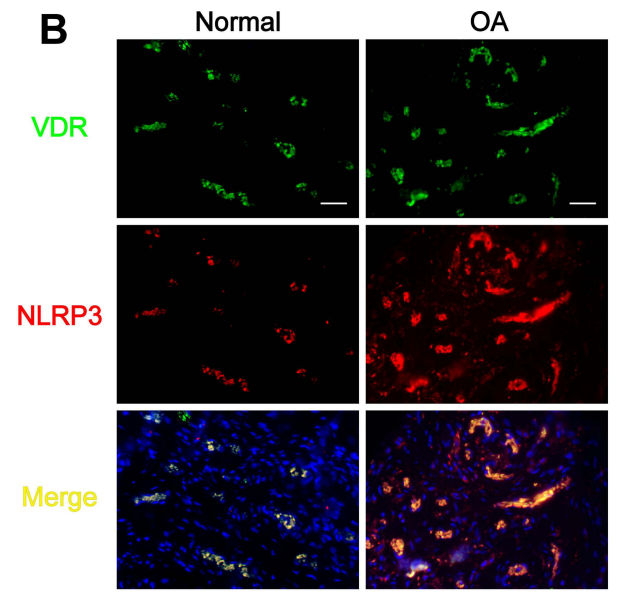

D

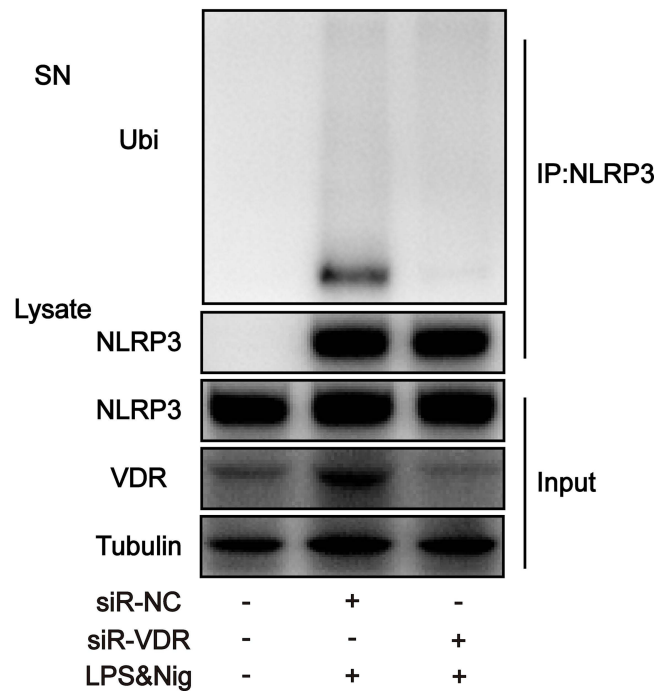

Figure 3 VDR directly interacts with NLRP3 and inhibits NLRP3 activity by increasing NLRP3 ubiquitination level. (A) Cell lysates were immunoprecipitated (IP) and immunoblotted (IB) with the indicated antibodies in each group. (B) Colocalization of VDR and NLPR3 in synovial sections of osteoarthritis patients and normal subjects as determined by immunofluorescence. Scale bar $=100 \mu \mathrm{m}$. (C) Western blot analysis of IL-I $\beta$ and cleaved caspase-I levels in culture SN and pro-IL-I $\beta$, pro-caspase-I and VDR levels in cell lysates in each group. (D) NLRP3 ubiquitination was analyzed in THP-I cells with different treatments.

with the VDR knock down group, indicating that VDR does play its anti-inflammatory role by regulating NLRP3 (Figure 3C). These findings indicate that VDR is implicated in NLRP3 activation.

Previous studies report that ubiquitination of NLRP3 is a key step in repressing NLRP3 activation. ${ }^{47}$ Therefore, the role of VDR in regulating NLRP3 ubiquitination and inhibiting its activation was explored. The findings indicated VDR knockdown significantly reduced ubiquitination level of NLRP3 compared with the LPS \& Nig induced negative control (NC) group (Figure 3D). This finding indicated increased NLRP3 activation, which is consistent with the changes in NLRP3 downstream signals presented in Figure 3C. In summary, these findings indicate that VDR directly interacts with NLRP3 and promotes
NLRP3 ubiquitination level and activation, subsequently reducing caspase-1 activity and IL-1 $\beta$ secretion in macrophages.

\section{Macrophage VDR Partly Reverses Decrease in Chondrocyte Proliferation Rate Caused by NLRP3 Activation}

Although the relationship between VDR and NLRP3 has been elucidated in the above studies, this interaction of macrophages and how it affects chondrocytes should be explored. Therefore, a co-culture system of THP-1 and human primary chondrocytes was established for the subsequent experiments (Figure 4). EdU flow cytometry analysis findings showed no significant changes in 


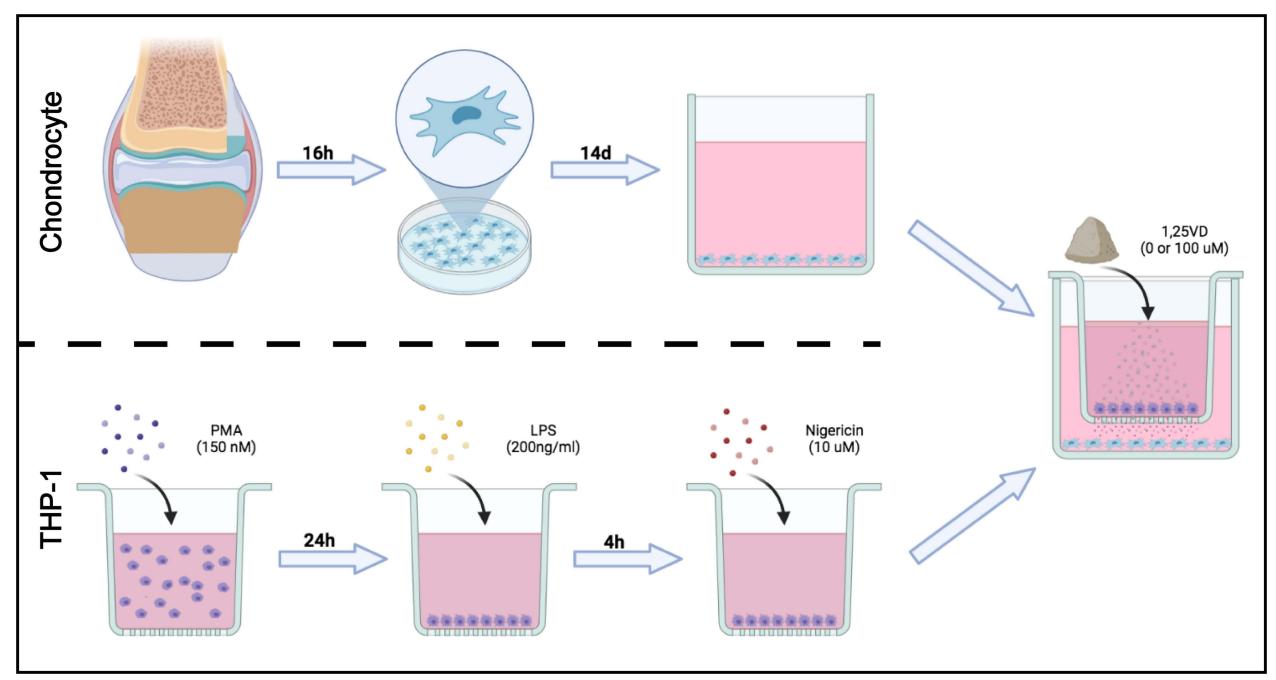

Figure 4 THP-I and chondrocyte co-culture system establishment model diagram.

proliferation rate of chondrocytes cultured alone with or without LPS \& Nig stimulation (Figure 5A and C). However, when chondrocytes were co-cultured with THP-1 without knockdown of VDR (MФ-NC ${ }^{\mathrm{KD}}$ ), the proliferation rate of chondrocytes in the co-culture system treated with LPS \& Nig was significantly lower compared with that in the co-culture system without LPS \& Nig (Figure 5A and $\mathrm{C}$ ). The findings showed that chondrocyte proliferation rate was significantly reduced when the chondrocytes were co-cultured with THP-1 with knockdown VDR (M $\Phi-\mathrm{VDR}^{\mathrm{KD}}$ ) compared with that of chondrocytes co-cultured with MФ-NC ${ }^{\mathrm{KD}}$ after addition of LPS \& Nig stimulated co-culture system. These findings were consistent with EdU fluorescence results (Figure 5B and D). The findings indicate that VDR in THP-1 promotes proliferation of chondrocytes.

\section{Macrophage VDR Partly Abrogates Increase in Chondrocyte Apoptosis Rate Caused by NLRP3 Activation}

Apoptosis rate of chondrocytes in each group was determined through the Annexin V-FITC/PI Apoptosis assay. The result showed no difference between the apoptosis rate of chondrocytes in the group of chondrocytes cultured alone treated with LPS \& Nig treatment and the group without LPS \& Nig treatment (Figure 5E and G). Apoptosis rate of chondrocytes in the co-culture system stimulated by LPS \& Nig was significantly increased for chondrocytes were co-cultured with $\mathrm{M} \Phi-\mathrm{NC}^{\mathrm{KD}}$ or $\mathrm{M} \Phi-$ $\mathrm{VDR}^{\mathrm{KD}}$. The findings showed that apoptosis rate of chondrocytes co-cultured with $\mathrm{M} \Phi-\mathrm{VDR}^{\mathrm{KD}}$ was significantly higher compared with that of chondrocytes cocultured with $\mathrm{M} \Phi-\mathrm{NC} \mathrm{KD}^{\mathrm{KD}}$ after LPS \& Nig treatment (Figure 5E and $\mathrm{G}$ ). Consistent findings were observed for the apoptotic rate of chondrocyte using the TUNEL Bright Green Apoptosis Detection Kit (Figure 5F and $H)$. These findings indicate that the interaction between VDR and NLRP3 in THP-1 can inhibit apoptosis of chondrocytes.

\section{Macrophage VDR Partly Alleviates the Decrease in Chondrocyte Migration Caused by NLRP3 Activation}

In addition, transwell assay and cell scratch area healing assay were conducted to detect the migration ability of chondrocytes in each group. The results of transwell assay showed no significant difference in the number of migrating chondrocytes cultured alone with and without LPS \& Nig treatment. However, cell migration rate decreased significantly when chondrocytes were co-cultured with $\mathrm{M} \Phi-\mathrm{NC} C^{\mathrm{KD}}$ or $\mathrm{M} \Phi-\mathrm{VDR}^{\mathrm{KD}}$ after addition of LPS \& Nig to the co-culture system. Moreover, the migration rate of chondrocytes co-cultured with $\mathrm{M} \Phi-\mathrm{VDR}^{\mathrm{KD}}$ was significantly lower compared with that of $\mathrm{M} \Phi-\mathrm{NC} \mathrm{KD}^{\mathrm{KD}}$ group induced by LPS \& Nig (Figure 6A and C). Consistent findings were obtained from the cell scratch healing assay (Figure 6B and D). These findings indicate that the interaction between VDR and NLRP3 in THP-1 promotes migration of chondrocytes. 
A

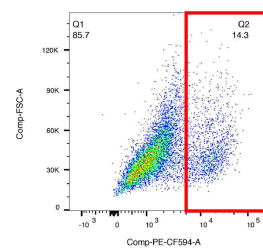

B

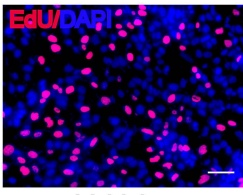

Vehicle

C

E

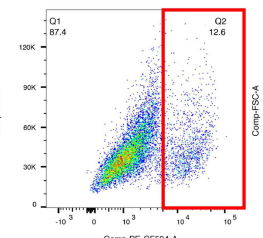

Vehicle

MФ-NC ${ }^{\mathrm{KD}}$

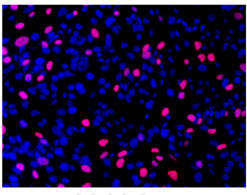

Vehicle

MФ-NC ${ }^{K D}$

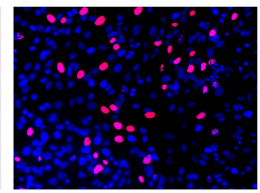

LPS\&Nig

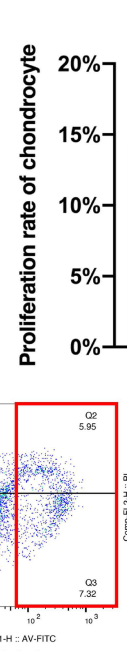

F Vehicle

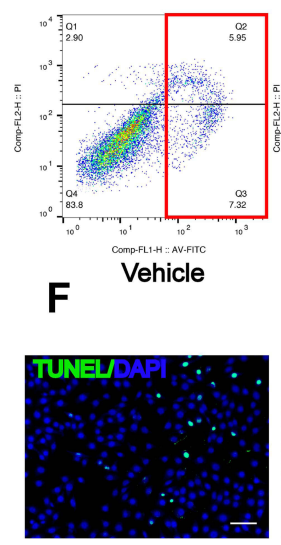

Vehicle

G
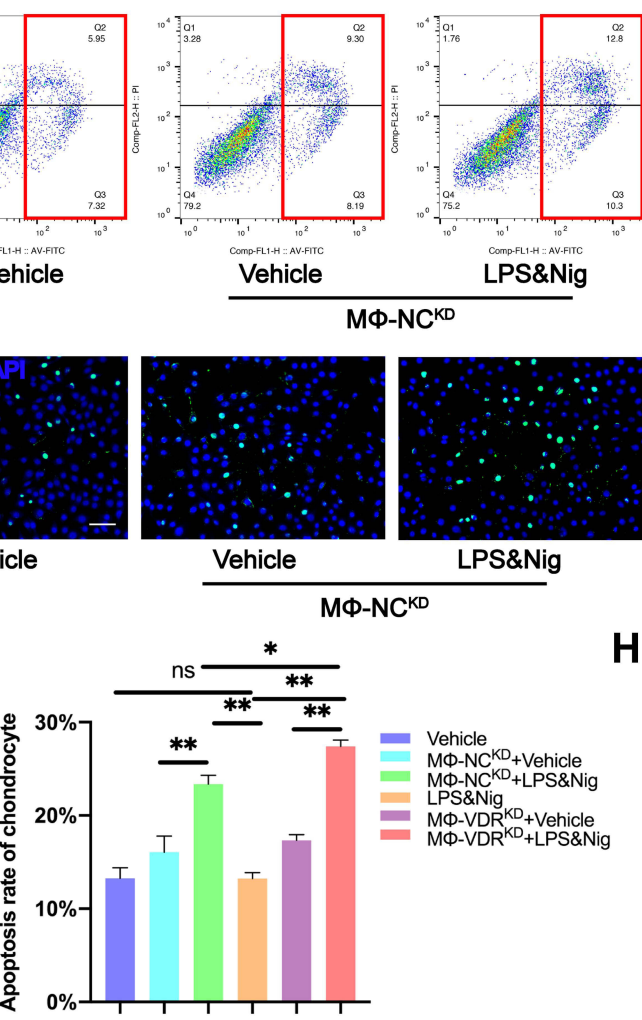

H

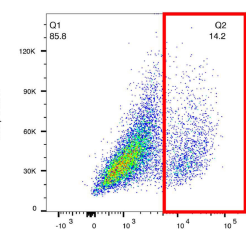

LPS\&Nig

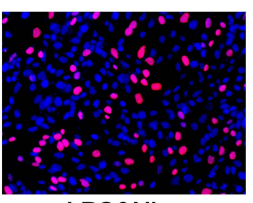

LPS\&Nig

D
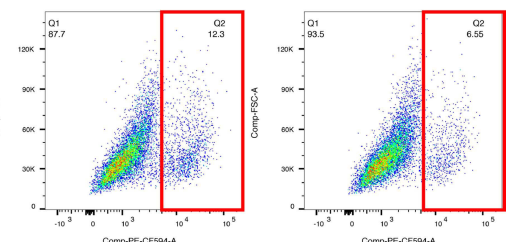

Vehicle

LPS\&Nig

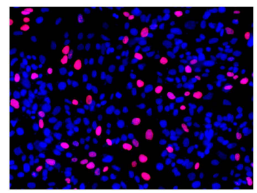

Vehicle

$M \Phi-V D R^{K D}$

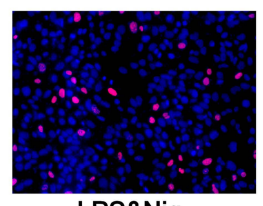

LPS\&Nig
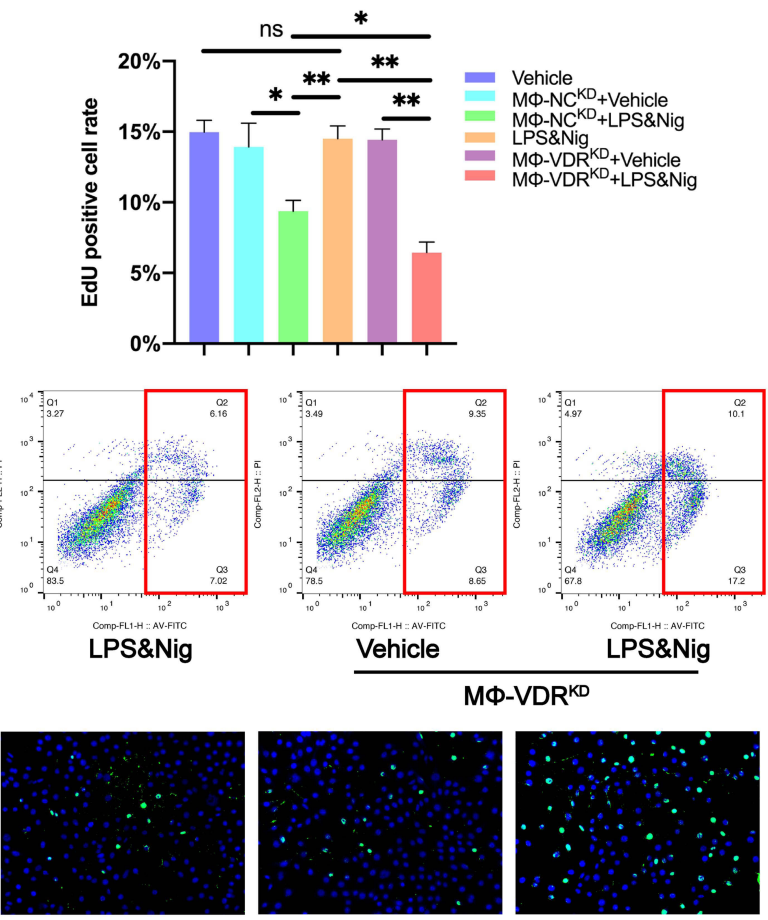

Vehicle

LPS\&Nig
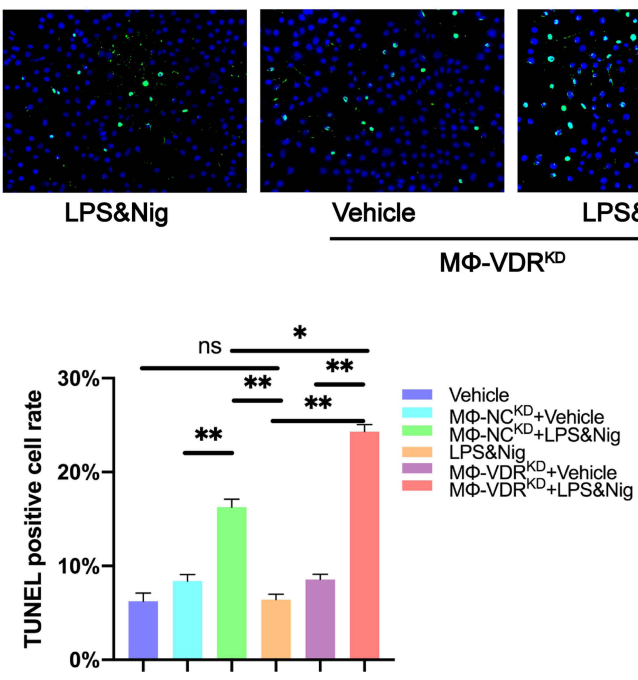

Figure 5 Macrophage VDR partly reverses decrease in chondrocyte proliferation rate and increase in chondrocyte apoptosis rate caused by NLRP3 activation. (A) Proliferation rate of chondrocytes was determined by flow cytometry analysis after EdU assay. (B) Proliferation rate of chondrocyte as determined by immunofluorescence. Scale bar $=200 \mu \mathrm{m}$. (C) Flow cytometry analysis of results from EdU assay $(n=3$, one-way ANOVA). (D) EdU positive cell rate of each group ( $n=3$, one-way ANOVA). (E) TUNEL assay for apoptosis rate of chondrocytes determined by flow cytometry analysis. (F) Apoptosis rate of chondrocytes as determined by immunofluorescence. Scale bar $=200 \mu \mathrm{m}$. (G) Flow cytometry analysis of TUNEL assay results $(n=3$, one-way ANOVA). $(\mathbf{H})$ TUNEL positive cell rate for each group $(n=3$, one-way ANOVA). Data are presented as mean $\pm \mathrm{SD}$. ns, no significance; ${ }^{*} \mathrm{p}<0.05$; ${ }^{* *} \mathrm{p}<0.01$. 


\section{A}

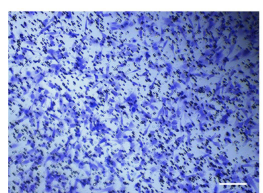

Vehicle

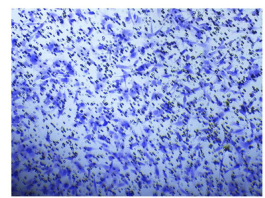

Vehicle

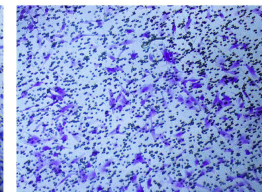

LPS\&Nig

B

$$
\text { MФ-NC }{ }^{K D}
$$
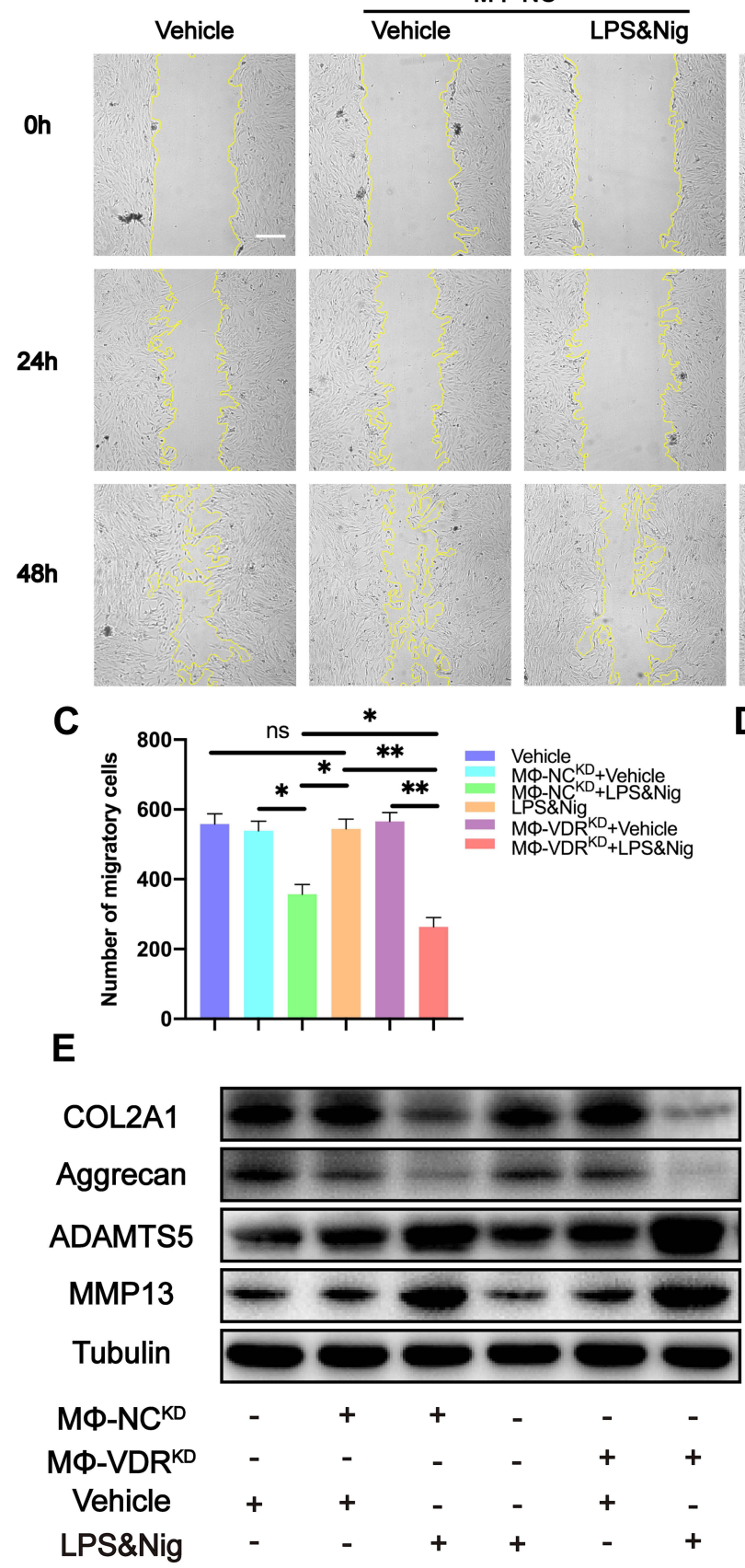

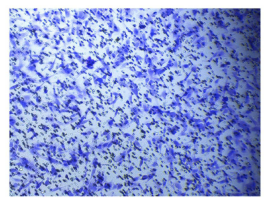

LPS\&Nig

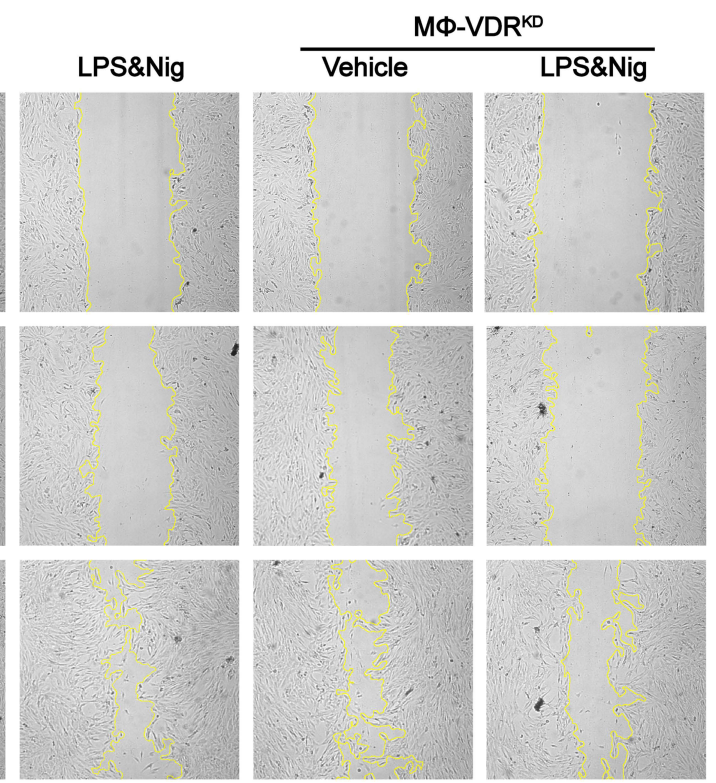

D
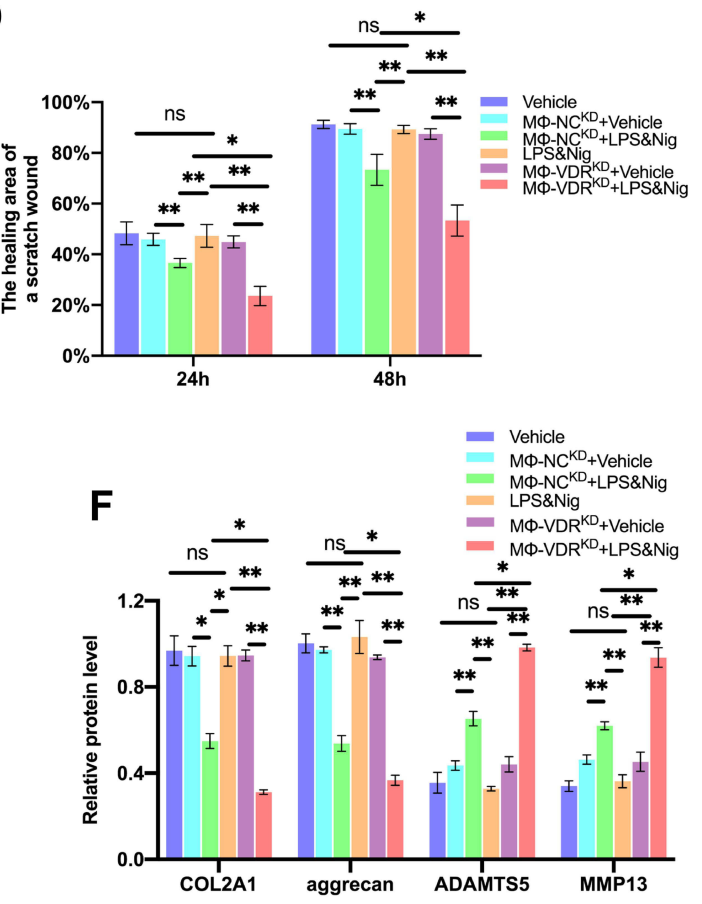

Figure 6 Macrophage VDR partly reverses the decrease in chondrogenic migration and inhibits the degradation of ECM proteins and the increase of ECM degradationassociated proteases in chondrocytes caused by NLRP3 activation. (A) Migration of chondrocytes was observed using transwell assay. Scale bar $=200 \mu \mathrm{m}$. (B) Images of scratch assays on chondrocytes were obtained under a light field of microscope. (C) The number of migratory cells was calculated and analyzed in each group ( $\mathrm{n}=3$, oneway ANOVA). Scale bar $=200 \mu \mathrm{m}$. (D) The healing area of a scratch wound was analyzed in each group $(n=3$, one-way ANOVA). (E) Western blot analysis for COL2AI, aggrecan, MMPI3 and ADAMTS5. (F) Quantification of the density of immunoreactive bands normalized to tubulin in each group $(n=3$, one-way ANOVA). Data are presented as mean \pm SD. ns, no significance; ${ }^{*} \mathrm{p}<0.05$; ${ }^{*} \mathrm{p}<<0.01$. 
Macrophage VDR Inhibits Degradation of ECM Proteins (COL2AI and Aggrecan) and Increases Levels of ECM Degradation-Associated Proteases (MMPI 3 and ADAMTS5) Caused by NLRP3 Activation in Chondrocytes

Expression levels of ECM proteins (COL2A1 and aggrecan) and ECM degradation-associated proteases (MMP13 and ADAMTS5) were explored by Western blot. The findings showed that addition of LPS \& Nig to chondrocytes cultured alone did not cause significant differences in the expression of COL2A1, aggrecan, MMP13 and ADAMTS5 proteins (Figure 6E). However, expression of ECM proteins (COL2A1 and aggrecan) was significantly downregulated whereas expression of ECM degradation-associated proteases (MMP13 and ADAMTS5) was significantly upregulated after treatment of the co-culture system with LPS \& Nig. Expression of ECM proteins (COL2A1 and aggrecan) in $\mathrm{M} \Phi-\mathrm{VDR}^{\mathrm{KD}}$ group with LPS \& Nig treatment was significantly downregulated compared with the levels in chondrocytes co-cultured with $\mathrm{M} \Phi-\mathrm{NC} \mathrm{KD}^{\mathrm{KD}}$, whereas expression of ECM degradation-associated proteases (MMP13 and ADAMTS5) was significantly upregulated (Figure 6E and F). These findings indicate that the interaction between VDR and NLRP3 in THP-1 inhibits degradation of ECM proteins (COL2A1 and aggrecan) and abrogates increase of ECM degradationassociated increased expression of proteases (MMP13 and ADAMTS5) induced by NLRP3 activation in chondrocytes.

\section{VDR Inhibits Secretion of Inflammatory Factors by Macrophages}

The effects reported above may be associated with inflammatory factors secreted by THP-1. Therefore, ELISA kits were used to determine levels of major inflammatory factors in each group. The findings showed that the levels of IL-1 $\beta$ and IL-18 in $M \Phi-$ $\mathrm{VDR}^{\mathrm{KD}}$ group were significantly increased after the LPS \& Nig treatment compared with the levels in the $\mathrm{M} \Phi-\mathrm{NC}^{\mathrm{KD}}$ group (Figure S1 A), whereas the level of TNF- $\alpha$ was not significantly different (Figure S1 A). These findings indicate that VDR inhibits NLRP3 activity thus reducing secretion of IL-1 $\beta$ and IL-18.

\section{I,25VD Enhances Ubiquitination Level of} NLRP3 by Modulating Interaction Between VDR and NLPR3

We used CCK-8 kit to explore the cytotoxicity of $1,25 \mathrm{VD}$, and the results showed that when 1,25VD concentration was $0-100 \mathrm{uM}$, there was no significant decrease on the survival rate of THP- 1 cells, and when 1,25VD concentration was above $200 \mathrm{uM}$, the survival rate of THP-1 cells decreased significantly (Figure S1 B). Therefore, we controlled the concentration range of the 1,25VD dose effect experiment between $0-100 \mathrm{uM}$. The findings showed that 1,25VD inhibited NLRP3 activation and expression of downstream caspase- 1 and IL-1 $\beta$ after treatment of THP1 with LPS \& Nig in a dose-dependent manner (Figure $\underline{\text { S1 C). }}$.

In order to further explore the mode of action of $1,25 \mathrm{VD}$, we used siRNA to specifically knock down the VDR in THP-1. It was found that there was no significant change in the secretion of caspase-1 and IL-1 $\beta$ with or without $1,25 \mathrm{VD}$, and the secretion of caspase- 1 and IL-1 $\beta$ increased significantly (Figure 7A), indicating that 1,25VD plays an anti-inflammatory role through VDR. When we knocked down the NLRP3 specificity of THP-1 with siRNA, there was no significant change in the secretion of caspase-1 and IL-1 $\beta$ with or without $1,25 \mathrm{VD}$ (Figure 7A), indicating that $1,25 \mathrm{VD}$ regulates the activity of NLRP3 through VDR, so as to play an antiinflammatory role.

Further analysis was performed to verify that the 1,25VD modulated interaction between VDR and NLRP3 ubiquitination. The findings showed that 1,25VD increased ubiquitination level of NLRP3 after LPS \& Nig treatment. However, this effect was abrogated by VDR knockdown (Figure 7B). These findings indicate that 1,25VD enhances NLRP3 ubiquitination through VDR and inhibits NLRP3 mediated macrophage inflammation response.

\section{I,25VD Enhances the Inhibitory Effect of VDR on Inhibition of Chondrocyte Proliferation Caused by NLRP3 \\ Activation}

The THP-1 and chondrocytes co-culture system was established to explore the effects of $1,25 \mathrm{VD}$ on proliferation of chondrocytes. Flow cytometry results showed that addition of $1,25 \mathrm{VD}$ significantly increased the proliferation rate of chondrocytes when chondrocytes were co-cultured with 
A

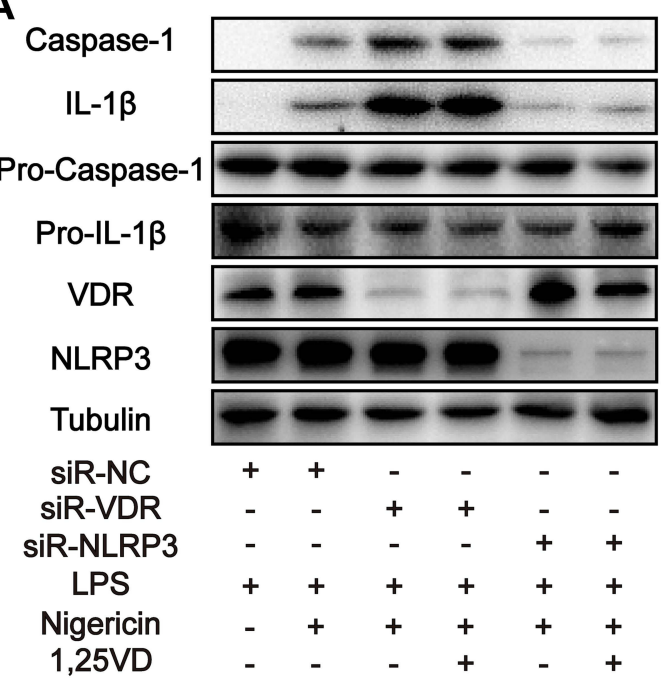

B SN

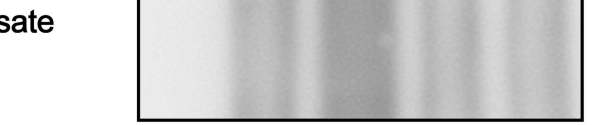

P:NLRP3

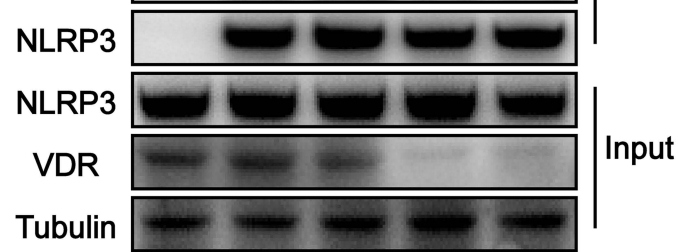

C

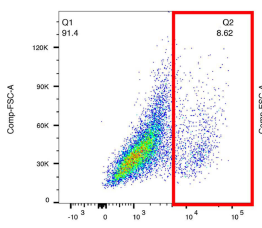

MФ-NC ${ }^{\mathrm{KD}}+$ Vehicle

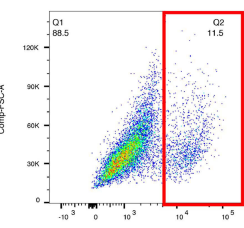

MФ-NC ${ }^{K D}+1,25 V D$

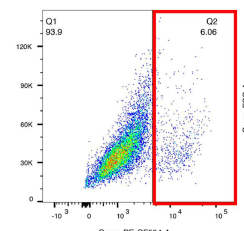

MФ-VDR ${ }^{\text {KDD }}+$ Vehicle

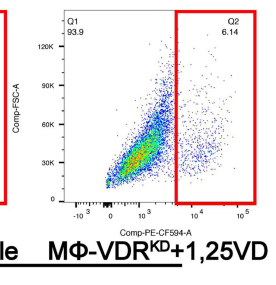

D

E

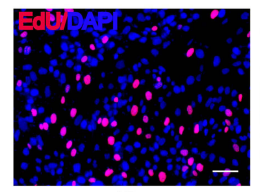

$\mathrm{M} \Phi-\mathrm{NC}^{\mathrm{KD}}+$ Vehicle

G

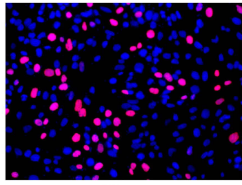

$\mathrm{M} \Phi-N C^{K D}+1,25 V D$

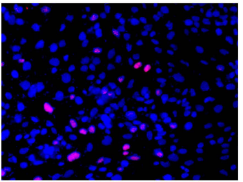

MФ-VDR ${ }^{\mathrm{KD}}+$ Vehicle

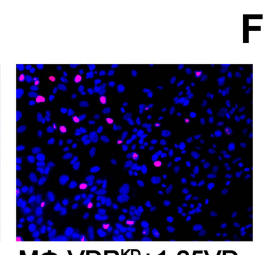

$M \Phi-V D R^{K D}+1,25 V D$

F
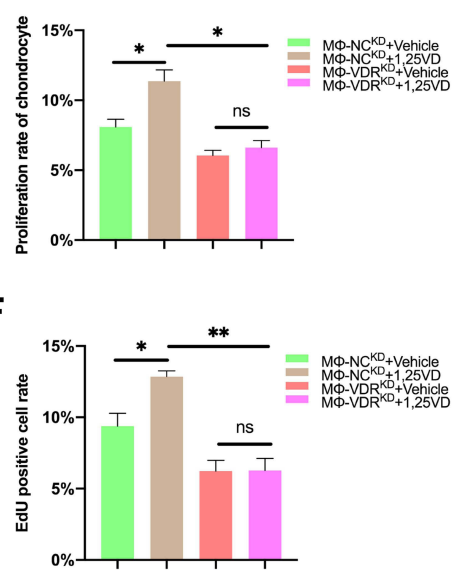

H

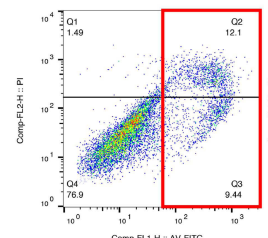

MФ-NC $\stackrel{\text { KD }+ \text { Vehicle }}{ }$
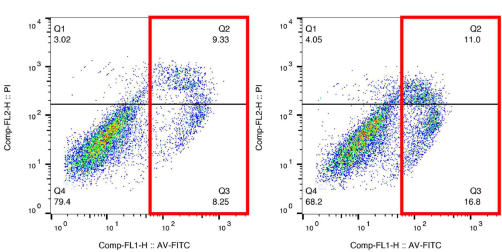

$M \Phi-N C^{K D}+1,25 V D$
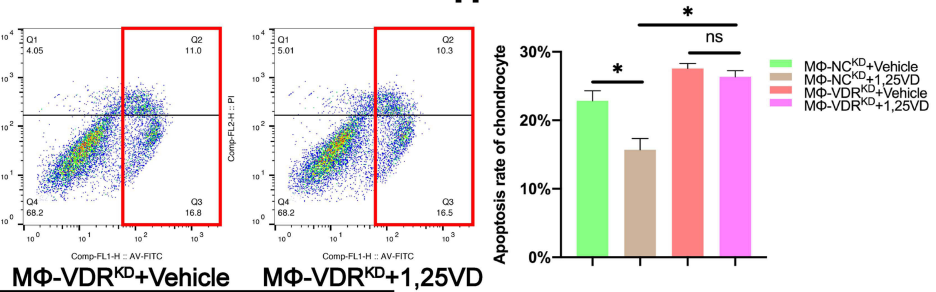

I LPS\&Nig

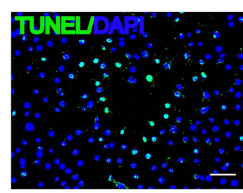

$\mathrm{M} \Phi-\mathrm{NC}^{\mathrm{KD}}+$ Vehicle

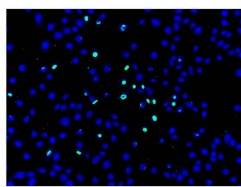

$\mathrm{M} \Phi-\mathrm{NC}^{\mathrm{KD}}+1,25 \mathrm{VD}$

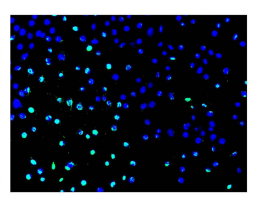

$M \Phi-V D R^{K D}+$ Vehicle

$M \Phi-V^{2}{ }^{\mathrm{KD}}+1,25 \mathrm{VD}$

\section{$\mathrm{J}$}

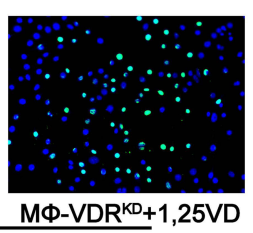

$M \Phi-V D R^{K D}+1,25 V D$

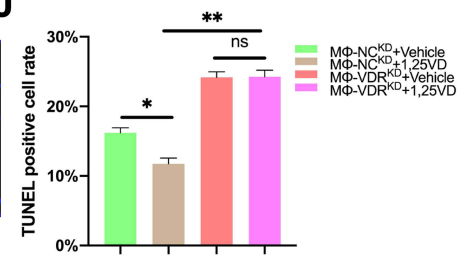

Figure 7 I,25VD enhances the inhibitory effect of VDR on the decrease of chondrocyte proliferation rate and the increase of chondrocyte apoptosis rate caused by NLRP3 activation (A) Western blot analysis of IL-I $\beta$ and cleaved caspase-I in SN and pro-IL-I $\beta$, pro-caspase-I, and VDR in cell lysates were detected in each group. (B) NLRP3 ubiquitination was analyzed in THP-I cells with different treatments. (C and D) EdU assay for proliferation rate of chondrocytes was determined by flow cytometry analysis and the statistical results of flow cytometry analysis for EdU assay ( $n=3$, one-way ANOVA). (E and $\mathbf{F}$ ) The proliferation rate of chondrocytes was observed by immunofluorescence and the EdU positive cell rate was analyzed in each group ( $n=3$, one-way ANOVA). Scale bar $=200 \mu \mathrm{m}$. (G and $\mathbf{H})$ TUNEL assay for apoptosis rate of chondrocytes was determined by flow cytometry analysis and the statistical results of flow cytometry analysis for TUNEL assay ( $\mathrm{n}=3$, one-way ANOVA). (I and $\mathbf{J})$ The apoptosis rate of chondrocytes was observed by immunofluorescence and the TUNEL positive cell rate was analyzed in each group $(n=3$, one-way ANOVA). Scale bar $=$ $200 \mu \mathrm{m}$. Data are presented as mean \pm SD. ns, no significance; *p $<0.05 ;{ }^{* *} \mathrm{p}<0.01$. 
$\mathrm{M} \Phi-\mathrm{NC}^{\mathrm{KD}}$ (Figure 7C and D). However, addition of $1,25 \mathrm{VD}$ did not increase proliferation rate of chondrocytes when chondrocytes were co-cultured with $\mathrm{M} \Phi-\mathrm{VDR}^{\mathrm{KD}}$ (Figure 7C and D). These findings were consistent with EdU fluorescence results (Figure 7E and F). The findings indicate that $1,25 \mathrm{VD}$ plays an important role in enhancing chondrocyte proliferation through VDR.

\section{I,25VD Enhances Inhibitory Effect of VDR of Chondrocyte Apoptosis Caused by NLRP3 Activation}

Annexin V-FITC/PI Apoptosis Detection Kit and the TUNEL Bright Green Apoptosis Detection Kit were used to determine the apoptosis rate of chondrocytes in each group. Flow cytometry results showed that treatment with $1,25 \mathrm{VD}$ significantly decreased apoptosis rate of chondrocytes when chondrocytes were co-cultured with $\mathrm{M} \Phi-\mathrm{NC} \mathrm{KD}^{\mathrm{KD}}$ (Figure $7 \mathrm{G}$ and $\mathrm{H}$ ). However, addition of $1,25 \mathrm{VD}$ did not reduce apoptosis rate of chondrocytes when chondrocytes were co-cultured with $\mathrm{M} \Phi-\mathrm{VDR}^{\mathrm{KD}}$ (Figure $7 \mathrm{G}$ and $\mathrm{H}$ ). TUNEL fluorescence results were consistent with flow cytometry results (Figure 7I and J). These findings indicate that $1,25 \mathrm{VD}$ plays a vital role in inhibiting chondrocyte apoptosis through VDR.

\section{I,25VD Enhances the Inhibitory Effect of VDR on the Decrease of Chondrocyte Migration Caused by NLRP3 Activation}

Transwell assay and cell scratch area healing assay were performed to determine migration ability of each group of chondrocytes. The number of migrating chondrocytes was significantly increased after treatment with 1,25VD when chondrocytes were co-cultured with $\mathrm{M} \Phi-\mathrm{NC}{ }^{\mathrm{KD}}$ (Figure 8A and B). The effect of 1,25VD was abrogated by VDR knockdown in THP-1 cells (Figure 8A and B). This finding indicates that $1,25 \mathrm{VD}$ promotes chondrocyte migration through VDR, and similar results were obtained from the cell scratch area healing assay (Figure $8 \mathrm{C}$ and D).

\section{I,25VD Enhances Inhibitory Effect of VDR on Decrease of ECM Proteins (COL2AI and Aggrecan) and Increase in ECM Degradation-Associated Proteases (MMPI 3 and ADAMTS5) Caused by NLRP3 Activation}

Western blot analysis was performed to explore the expression levels of related proteins in each group. The findings showed that the expression levels of ECM proteins (COL2A1 and aggrecan) increased significantly and that of ECM degradation-associated proteases (MMP13 and ADAMTS5) decreased significantly in chondrocytes co-cultured with $\mathrm{M} \Phi-\mathrm{NC}^{\mathrm{KD}}$ and treated with LPS \& Nig after treatment with 1,25VD (Figure 8E and F). Notably, this effect was abrogated by knockdown of VDR in THP-1 cells (Figure 8E and F). These findings indicated that 1,25VD upregulated expression of ECM proteins (COL2A1 and aggrecan) and downregulated expression of ECM degradation-associated proteases (MMP13 and ADAMTS5) through VDR.

\section{I,25VD Enhances Ability of VDR to Inhibit Secretion of Inflammatory Cytokines by Macrophages}

The above findings implied that $1,25 \mathrm{VD}$ may improve ubiquitination level of NLRP3 by modulating interaction between VDR and NLRP3, thus inhibiting secretion of inflammatory factors by macrophage ultimately playing a protective role on chondrocytes. Therefore, ELISA kits were used to determine the levels of major inflammatory factors in each group of cells. ELISA results showed that $1,25 \mathrm{VD}$ had no regulatory effect on TNF- $\alpha$ level (Figure $\underline{\mathrm{S} 2 \mathrm{D})}$. However, 1,25VD significantly reduced the levels of IL-1 $\beta$ and IL-18 in the MФ-NC ${ }^{\mathrm{KD}}$ group (Figure S2 D). However, the effect of $1,25 \mathrm{VD}$ on levels of inflammatory factors was abrogated knockdown of VDR in THP-1 cells (Figure S2 D).

\section{Discussion}

Osteoarthritis is the most prevalent chronic articular disease and is characterized by progressive degeneration of articular cartilage, as well as bone remodeling. ${ }^{48}$ Currently, a number of therapies are available for OA, including pharmacological and non-pharmacological approaches. $^{49-51}$ Previous clinical studies reported that vitamin $\mathrm{D}$ endocrine system, including vitamin $\mathrm{D}$ and its receptor VDR, are involved in cartilaginous metabolisms, which are associated with pathological changes of OA. ${ }^{52-54}$ However, the potential mechanisms have not been fully elucidated. Therefore, the current study explored the role of vitamin D in alleviation of pathological changes of OA in DMM-induced OA mice model. The findings showed protective effects of vitamin D against chondrogenic ECM degradation and osteophytes formation. Furthermore, degradation of ECM protein (COL2A1 
A

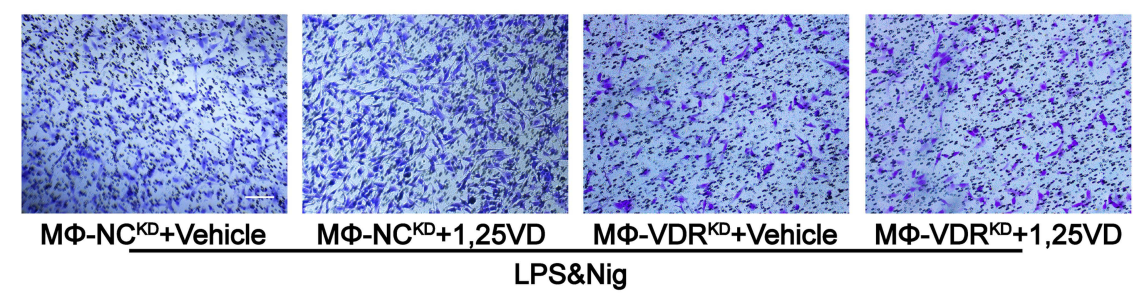

B

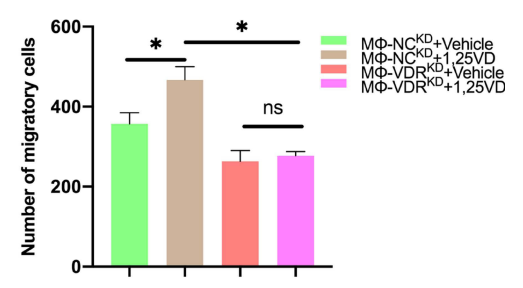

C

LPS\&Nig

$M \Phi-N C^{K D}+$ Vehicle $M \Phi-N C^{K D}+1,25 V D M \Phi-V D R^{K D}+$ Vehicle $M \Phi-V D R^{K D}+1,25 V D$
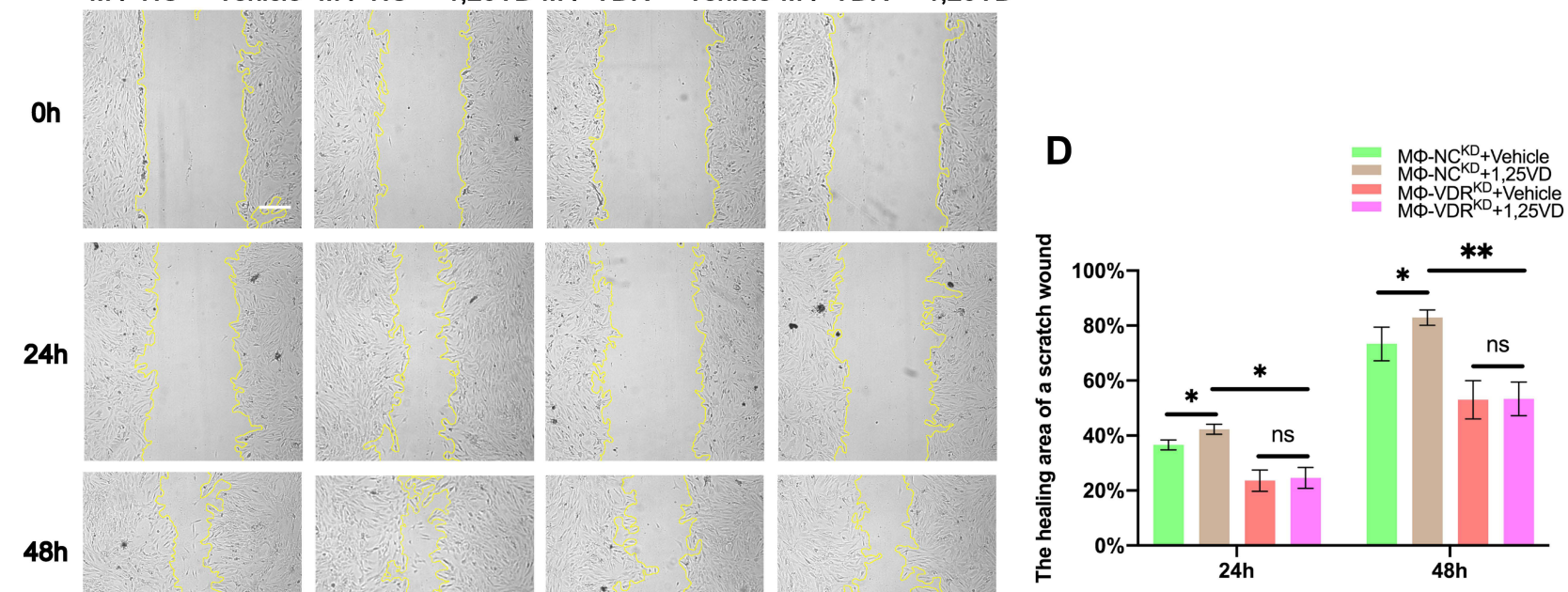

48h
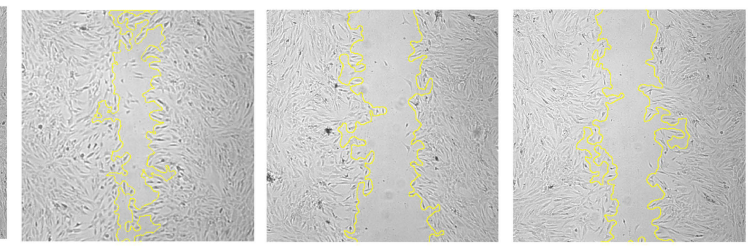

E
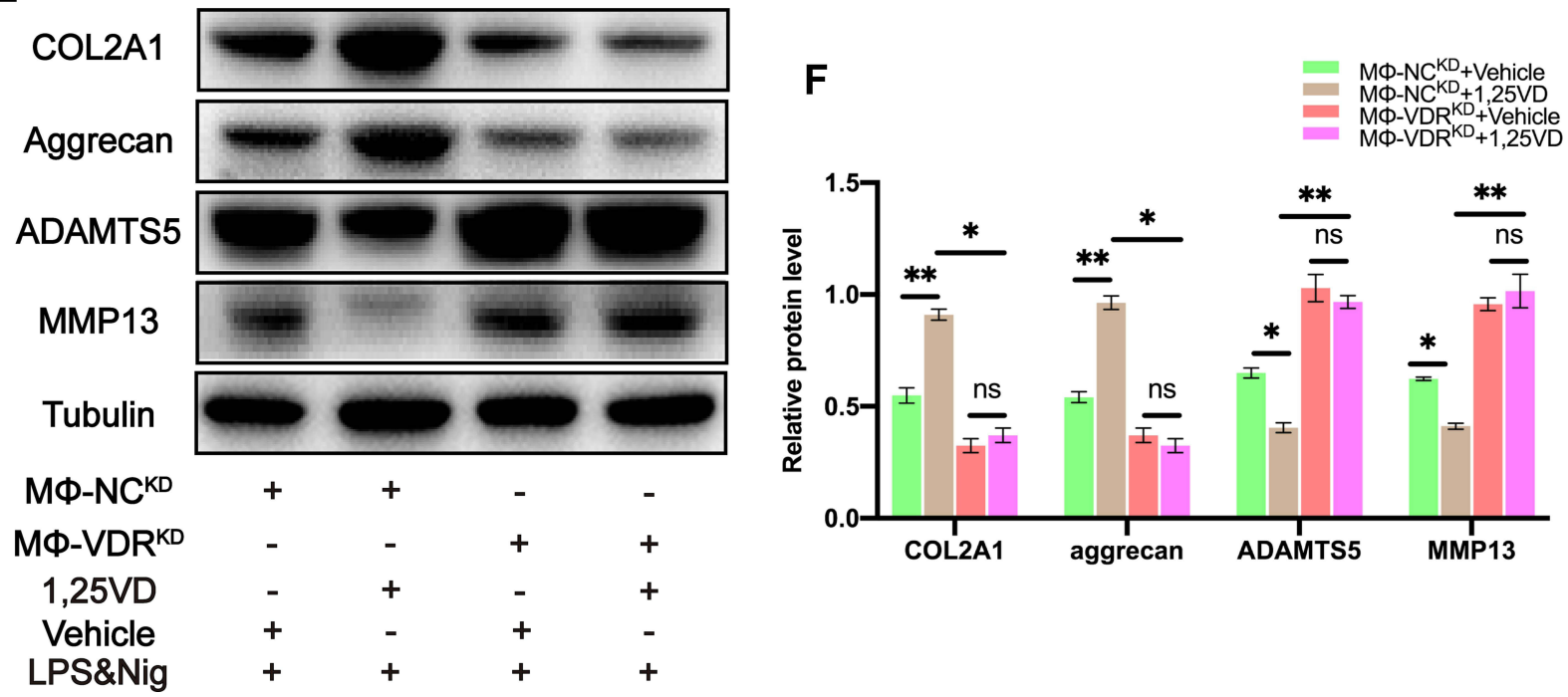

Figure 8 I,25VD enhances the inhibitory effect of VDR on the decrease of chondrocyte migration and the decrease of ECM proteins and the increase of ECM degradationassociated proteases caused by NLRP3 activation. (A and B) Migration of chondrocytes was observed and quantified by transwell assay and crystal violet was used to stain migrating chondrocytes $(n=3$, one-way ANOVA). Scale bar $=200 \mu \mathrm{m}$. (C and D) Images of scratch assays on chondrocytes were obtained under a light microscope and the healing area of a scratch wound was analyzed in each group ( $n=3$, one-way ANOVA). Scale bar $=200 \mu \mathrm{m}$. (E and F) Western blot analysis of COL2AI, aggrecan, ADAMTS5 and MMPI 3 in chondrocytes were detected and quantified in each group $(n=3$, one-way ANOVA). Data are presented as mean \pm SD. ns, no significance; *p <0.05; ** $p<0.0$ I.

and aggrecan) and abnormally increased expression of

ECM degradation-associated proteases (MMP13 and ADAMTS5) were reversed by treatment with vitamin
D. The findings on protective effects of vitamin $\mathrm{D}$ on inhibition of expression of ECM degradation-associated protease results were consistent with findings from 
a previous study. ${ }^{55}$ In addition, determination of levels of inflammatory cytokines in knee tissue of DMM-induced OA mice model in each group showed that vitamin D therapy significantly reduced IL-1 $\beta$ and IL-18 levels. Inflammatory response is a significant factor that initiates and aggravates progression of $\mathrm{OA}^{56}$ Previous studies reported that the interplay between inflammatory cytokines and chondrogenic ECM degradation plays a crucial role in onset and development of OA. ${ }^{57}$ These findings indicate that these symptoms may be caused by dysregulation of secretion of inflammatory cytokines. Further analyses were performed using $\mathrm{VDR}^{-/-}$mice and the findings showed that the protective effects of vitamin D on DMMinduced OA mice model were alleviated and the effects of vitamin D on the reduction of IL-1 $\beta$ and IL-18 levels were abrogated by VDR knockdown. This finding indicates that vitamin $\mathrm{D}$ exerts its joint protective effect by binding to VDR.

Vitamin D can reduce the levels of IL-1 $\beta$ and IL-18, which are closely related to NLRP3 activity. ${ }^{58}$ Notably, NLRP3 is a potential novel biomarker for OA and is implicated in induction of secretion of IL-1 $\beta$ and IL-18, which promote cartilage degeneration and synovial inflammation. ${ }^{45}$ This indicates that vitamin D may regulate NLRP3 activity by binding to VDR. It has previously been reported that VDR can affect NLRP3 in BMDM. ${ }^{46}$ The interaction between NLRP3 and VDR in THP-1 was further investigated by co-immunoprecipitation and immunofluorescence colocalization. Previous studies reported that ubiquitination is a key step in inhibition of NLPR3 activation. ${ }^{47}$ The findings of the current study showed that VDR increases ubiquitination level of NLRP3, thus reducing levels of IL-1 $\beta$ and IL-18 secreted by macrophages.

Macrophages are implicated in cartilage regeneration and present a potential target for protection of cartilage degradation. $^{23}$ Moreover, chondrocytes, as the sole cell type present within cartilage, play important roles in maintenance of tissue homeostasis. ${ }^{59}$ Therefore, further analysis was performed to explore whether VDR and NLRP3 interaction in macrophages affected chondrocytes by establishing a co-culture system between THP-1 and chondrocytes. In the co-culture system, the proliferation and migration ability of chondrocytes co-cultured with $\mathrm{M} \Phi-$ $\mathrm{VDR}^{\mathrm{KD}}$ were significantly lower and the apoptosis rate was significantly higher compared with that of chondrocytes cocultured with MФ-NC ${ }^{\mathrm{KD}}$ after NLRP3 activation. Moreover, expression level of ECM degradationassociated proteases (MMP13 and ADAMTS5) was significantly higher whereas expression level of ECM protein (COL2A1 and aggrecan) was significantly decreased in chondrocytes co-cultured with $\mathrm{M} \Phi-\mathrm{VDR}^{\mathrm{KD}}$ compared with the levels in the chondrocytes co-cultured with $\mathrm{M} \Phi-\mathrm{NC}{ }^{\mathrm{KD}}$. The levels of inflammatory cytokines in each group were explored and the findings showed that LPS \& Nig stimulation significantly increased the ability of $M \Phi-V D R^{\mathrm{KD}}$ to secrete IL-1 $\beta$ and IL-18 compared with that of MФ-NC ${ }^{\mathrm{KD}}$. These findings indicate that VDR plays a protective role in chondrocytes by increasing NLRP3 ubiquitination level, inhibiting NLRP3 activity, and reducing secretion of inflammatory cytokines such as IL-1 $\beta$ and IL-18.

These results implied that vitamin $\mathrm{D}$ exerts its antiarthritic effect by regulating NLRP3 ubiquitination level through VDR in vivo. Therefore, different concentrations of $1,25 \mathrm{VD}$ were added to THP-1 cells and the findings showed that IL- $1 \beta$ and caspase- 1 levels in THP-1 cells were positively correlated with the concentration of 1,25VD. Further analysis showed that 1,25VD significantly improved ubiquitination level of NLRP3, and this effect was abrogated by VDR knockdown. This finding indicatesd that $1,25 \mathrm{VD}$ plays a protective role by regulating NLRP3 ubiquitination level by binding to VDR. Furthermore, we found 1,25VD inhibited decrease of chondrocyte proliferation and migration ability and increased apoptosis rate induced by NLRP3 activation in the co-culture system. Moreover, 1,25VD inhibited degradation of ECM proteins (COL2A1 and aggrecan) and increased levels of ECM degradationassociated proteases (MMP13 and ADAMTS5) induced by macrophage activation. ELISA analysis was performed to explore inflammatory factors in each group, and the findings showed that 1,25VD inhibit secretion of IL-1 $\beta$ and IL-18. However, these 1,25VD effects were not observed after knockdown of VDR in THP-1 cells. These findings indicate that $1,25 \mathrm{VD}$ enhances the ability of VDR to increase ubiquitination level of NLRP3 and inhibits secretion of IL-1 $\beta$ and IL-18, thus protecting chondrocytes.

Osteoarthritis involves all joint compartments but this study only investigates the interaction of immune cells and chondrocytes. In summary, the findings of the current study show that $1,25 \mathrm{VD}$ ameliorates knee OA by modulating interaction between VDR and NLRP3 in macrophages, resulting in inhibition of NLRP3 activation and downstream cytokine (IL-1 $\beta$ and IL-18) secretion. As a result, it promotes proliferation and migration of chondrocytes, inhibits apoptosis of 


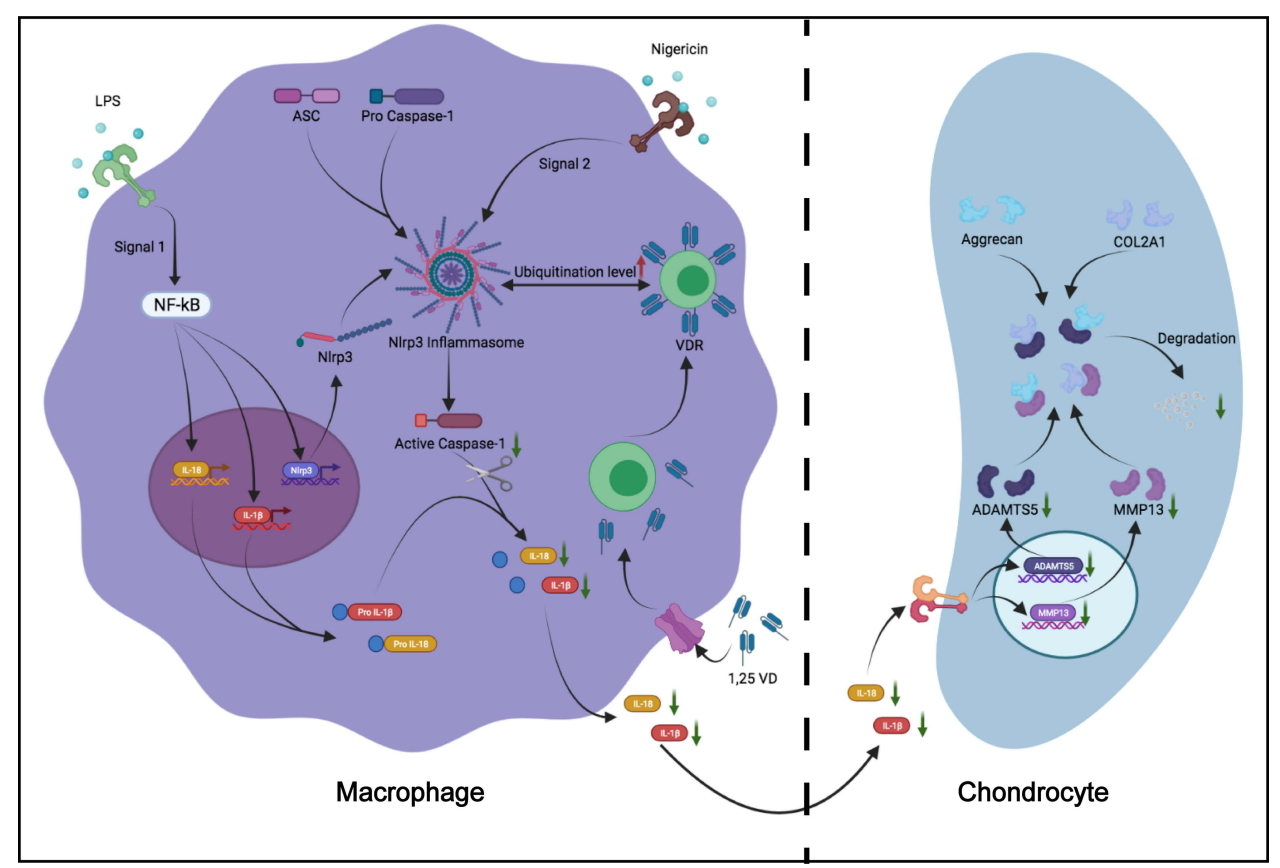

Figure 9 Model of I,25VD targeting VDR prevents the degradation of chondrogenic ECM by regulating the activation of NLRP3 and the secretion of inflammatory cytokines in macrophages.

chondrocytes, inhibits degradation of ECM protein (COL2A1 and aggrecan) and increases levels of ECM degradation-associated proteases (MMP13 and ADAMTS5) (Figure 9). These findings imply that VDR in macrophages is a promising target for OA treatment. In addition, the findings provide information on a novel molecular mechanism for effect of $1,25 \mathrm{VD}$ in $\mathrm{OA}$.

\section{Abbreviations}

OA, osteoarthritis; ECM, extracellular matrix; IL-1 $\beta$, interleukin-1 $\beta$; IL-18, interleukin-18; 1,25VD, 1,25dihydroxyvitamin D; DMM, destabilization of the medial meniscus; VDR, vitamin D receptor; NLRP3, nod-like receptor pyrin domain 3 inflammasome; COL2A1, type II collagen alpha 1; ADAMTS5, a disintegrin-like and metalloproteinase domain with thrombospondin-1 motifs 5; MMP13, matrix metalloproteinase 13; PMA, phorbol myristate acetate; Nig, nigericin; THA, total knee arthroplasty; OARSI, osteoarthritis research society international; $\mathrm{NC}$, negative control; $\mathrm{M} \Phi-\mathrm{VDR}^{\mathrm{KD}}$, knocking down VDR in THP-1.

\section{Data Sharing Statement}

All relevant data and materials are available from the authors upon reasonable request.

\section{Ethics Approval and Consent to Participate}

This study was approved by the ethics committee of The First Affiliated Hospital of Nanjing Medical University (No.2021-SR-027). Written informed consent was obtained from all participants included in the study.

All procedures performed in studies involving animals were conducted in accordance with the animal care and guidelines of The First Affiliated Hospital of Nanjing Medical University (No. IACUC-2006052).

\section{Acknowledgments}

We sincerely thank Professor Xiulan Sun for providing us with the experimental platform and technical guidance for this experiment, and we also thank Professor Yunzi Chen for providing us with $\mathrm{VDR}^{-/}$mice.

\section{Author Contributions}

All authors made a significant contribution to the work reported, whether that is in the conception, study design, execution, acquisition of data, analysis and interpretation, or in all these areas; took part in drafting, revising or critically reviewing the article; gave final approval of the version to be published; have agreed on the journal to which the article has been submitted; and agree to be accountable for all aspects of the work. 


\section{Funding}

This study was financially supported by the Natural Science Foundation of Jiangsu Province (Grant No. BK20191492).

\section{Disclosure}

All authors declare that they have no conflicts of interest to report.

\section{References}

1. Sharma L. Osteoarthritis of the knee. $N$ Engl J Med. 2021;384 (1):51-59. doi:10.1056/NEJMcp1903768

2. Lawrence RC, Felson DT, Helmick CG, et al. Estimates of the prevalence of arthritis and other rheumatic conditions in the United States. Part II. Arthritis Rheum. 2008;58(1):26-35. doi:10.1002/ art. 23176

3. Muthuri SG, McWilliams DF, Doherty M, et al. History of knee injuries and knee osteoarthritis: a meta-analysis of observational studies. Osteoarthritis Cartilage. 2011;19(11):1286-1293. doi:10. 1016/j.joca.2011.07.015

4. Agricola R, Heijboer MP, Roze RH, et al. Pincer deformity does not lead to osteoarthritis of the hip whereas acetabular dysplasia does: acetabular coverage and development of osteoarthritis in a nationwide prospective cohort study (CHECK). Osteoarthritis Cartilage. 2013;21(10):1514-1521. doi:10.1016/j.joca.2013.07.004

5. Yang S, Zusman N, Lieberman E, et al. Developmental dysplasia of the hip. Pediatrics. 2019;143(1):e20181147. doi:10.1542/peds.20181147

6. Agricola R, Heijboer MP, Bierma-Zeinstra SMA, et al. Cam impingement causes osteoarthritis of the hip: a nationwide prospective cohort study (CHECK). Ann Rheum Dis. 2013;72(6):918-923. doi:10.1136/annrheumdis-2012-201643

7. Zadpoor AA. Etiology of femoroacetabular impingement in athletes: a review of recent findings. Sports Med. 2015;45(8):1286-1293. doi:10.1007/s40279-015-0339-2

8. Sharma L, Chmiel JS, Almagor O, et al. The role of varus and valgus alignment in the initial development of knee cartilage damage by MRI: the MOST study. Ann Rheum Dis. 2013;72(2):235-240. doi:10.1136/annrheumdis-2011-201070

9. Felson DT, Niu J, Gross KD, et al. Valgus malalignment is a risk factor for lateral knee osteoarthritis incidence and progression: findings from the multicenter osteoarthritis study and the osteoarthritis initiative. Arthritis Rheum. 2013;65(2):787-791. doi:10.1002/ art. 37726

10. Harvey WF, Yang M, Cooke TD, et al. Association of leg-length inequality with knee osteoarthritis: a cohort study. Ann Intern Med. 2010;152(5):287-295. doi:10.7326/0003-4819-152-5-20100302000006

11. Hu J, Zhou J, Wu J, et al. Loganin ameliorates cartilage degeneration and osteoarthritis development in an osteoarthritis mouse model through inhibition of NF- $\mathrm{\kappa B}$ activity and pyroptosis in chondrocytes. J Ethnopharmacol. 2020;247:112261. doi:10.1016/j.jep.2019.112261

12. Duan A, Shen K, Li B, et al. Extracellular vesicles derived from LPS-preconditioned human synovial mesenchymal stem cells inhibit extracellular matrix degradation and prevent osteoarthritis of the knee in a mouse model. Stem Cell Res Ther. 2021;12(1):1-20. doi:10.1186/ s13287-021-02507-2.

13. Liao L, Zhang S, Gu J, et al. Deletion of Runx2 in articular chondrocytes decelerates the progression of DMM-induced osteoarthritis in adult mice. Sci Rep. 2017;7(1):2371. doi:10.1038/s41598-01702490-w
14. Rahmati M, Nalesso G, Mobasheri A, et al. Aging and osteoarthritis: central role of the extracellular matrix. Ageing Res Rev. 2017;40:20-30. doi:10.1016/j.arr.2017.07.004

15. Lü G, Li L, Wang B, et al. LINC00623/miR-101/HRAS axis modulates IL-1 $\beta$-mediated ECM degradation, apoptosis and senescence of osteoarthritis chondrocytes. Aging. 2020;12(4):3218-3237. doi:10.18632/ aging. 102801

16. Chen D, Shen J, Zhao W, et al. Osteoarthritis: toward a comprehensive understanding of pathological mechanism. Bone Res. 2017;5(1):16044. doi:10.1038/boneres.2016.44

17. Mead TJ, Apte SS. ADAMTS proteins in human disorders. Matrix Biol. 2018;71-72:225-239. doi:10.1016/j.matbio.2018.06.002

18. Hoshi H, Akagi R, Yamaguchi S, et al. Effect of inhibiting MMP13 and ADAMTS5 by intra-articular injection of small interfering RNA in a surgically induced osteoarthritis model of mice. Cell Tissue Res. 2017;368(2):379-387. doi:10.1007/s00441-016-2563-y

19. Luo P, Jiang C, Ji P, et al. Exosomes of stem cells from human exfoliated deciduous teeth as an anti-inflammatory agent in temporomandibular joint chondrocytes via miR-100-5p/mTOR. Stem Cell Res Ther. 2019;10(1):216. doi:10.1186/s13287-019-1341-7

20. Muttigi MS, Han I, Park HK, et al. Matrilin-3 role in cartilage development and osteoarthritis. Int J Mol Sci. 2016;17(4):590. doi:10.3390/ijms 17040590

21. Maldonado M, Nam J. The role of changes in extracellular matrix of cartilage in the presence of inflammation on the pathology of osteoarthritis. Biomed Res Int. 2013;2013:284873. doi:10.1155/ 2013/284873

22. Mabey T, Honsawek S. Cytokines as biochemical markers for knee osteoarthritis. World J Orthop. 2015;6(1):95-105. doi:10.5312/wjo.v6. i1.95

23. Fernandes TL, Gomoll AH, Lattermann C, et al. Macrophage: a potential target on cartilage regeneration. Front Immunol. 2020; 11:111. doi:10.3389/fimmu.2020.00111

24. Sanchez-Lopez E, Zhong Z, Stubelius A, et al. Choline uptake and metabolism modulate macrophage IL-1 $\beta$ and IL-18 production. Cell Metab. 2019;29(6):1350-1362.e7. doi:10.1016/j.cmet.2019.03.011

25. Hughes MM, O'Neill LAJ. Metabolic regulation of NLRP3. Immunol Rev. 2018;281(1):88-98. doi:10.1111/imr.12608

26. Yan Z, Qi W, Zhan J, et al. Activating Nrf2 signalling alleviates osteoarthritis development by inhibiting inflammasome activation. J Cell Mol Med. 2020;24(22):13046-13057. doi:10.1111/jcmm.15 905

27. Lamkanfi M, Dixit VM. Mechanisms and functions of inflammasomes. Cell. 2014;157(5):1013-1022. doi:10.1016/j.cell.2014.04.007

28. Chen X, Li Y, Zhang M, et al. Silencing LCK inactivates the NF$\kappa \mathrm{B}$ pathway to attenuate cartilage injury in osteoarthritis: an in vivo and in vitro study. $J$ Gene Med. 2021:e3387. doi:10. $1002 /$ jgm. 3387

29. Charoenngam N, Holick MF. Immunologic effects of Vitamin D on human health and disease. Nutrients. 2020;12(7):2097. doi:10.3390/ nu12072097

30. Sassi F, Tamone C, D'Amelio P. Vitamin D: nutrient, hormone, and immunomodulator. Nutrients. 2018;10(11):1656. doi:10.3390/nu101 11656

31. Kiourtzidis M, Kühn J, Brandsch C, et al. Markers indicating body Vitamin D stores and responses of liver and adipose tissues to changes in Vitamin D intake in male mice. Nutrients. 2020;12 (5):1391. doi:10.3390/nu12051391

32. Swanson KV, Deng M, Ting JP. The NLRP3 inflammasome: molecular activation and regulation to therapeutics. Nat Rev Immunol. 2019;19(8):477-489. doi:10.1038/s41577-019-0165-0

33. Bauernfeind FG, Horvath G, Stutz A, et al. Cutting edge: NF-kappaB activating pattern recognition and cytokine receptors license NLRP3 inflammasome activation by regulating NLRP3 expression. $J$ Immunol. 2009;183(2):787-791. doi:10.4049/ jimmunol.0901363 
34. Mao G, Zhang Z, Hu S, et al. Exosomes derived from miR-92a-3poverexpressing human mesenchymal stem cells enhance chondrogenesis and suppress cartilage degradation via targeting WNT5A. Stem Cell Res Ther. 2018;9(1):247. doi:10.1186/s13287-018-1004-0

35. Li Z, Zhang C, Wang Z, et al. Lipofectamine 2000/siRNA complexes cause endoplasmic reticulum unfolded protein response in human endothelial cells. $J$ Cell Physiol. 2019;234(11):21166-21181. doi:10.1002/jcp.28719

36. Liu L, Ishijima M, Kaneko H, et al. The MRI-detected osteophyte score is a predictor for undergoing joint replacement in patients with end-stage knee osteoarthritis. Mod Rheumatol. 2017;27(2):332-338. doi:10.1080/14397595.2016.1206509

37. Hu ZL, Sun T, Lu M, et al. Kir6.1/K-ATP channel on astrocytes protects against dopaminergic neurodegeneration in the MPTP mouse model of Parkinson's disease via promoting mitophagy. Brain Behav Immun. 2019;81:509-522. doi:10.1016/j.bbi.2019.07.009

38. Hu S, Zhang C, Ni L, et al. Stabilization of HIF-1 $\alpha$ alleviates osteoarthritis via enhancing mitophagy. Cell Death Dis. 2020;11 (6):481. doi:10.1038/s41419-020-2680-0

39. Yang G, Lee HE, Moon S-J, et al. Direct binding to NLRP3 pyrin domain as a novel strategy to prevent NLRP3-driven inflammation and gouty arthritis. Arthritis Rheumatol. 2020;72(7):1192-1202. doi:10.1002/art.41245

40. Zhang B-C, Nandakumar R, Reinert LS, et al. STEEP mediates STING ER exit and activation of signaling. Nat Immunol. 2020;21 (8):868-879. doi:10.1038/s41590-020-0730-5

41. Chen Y, Peng C, Chen J, et al. WTAP facilitates progression of hepatocellular carcinoma via m6A-HuR-dependent epigenetic silencing of ETS1. Mol Cancer. 2019;18(1):127. doi:10.1186/s12943-0191053-8

42. Li S, Ma F, Pang X, et al. Synthesis of chondroitin sulfate magnesium for osteoarthritis treatment. Carbohydr Polym. 2019;212:387-394. doi:10.1016/j.carbpol.2019.02.061

43. Shi Y, Hu X, Cheng J, et al. A small molecule promotes cartilage extracellular matrix generation and inhibits osteoarthritis development. Nat Commun. 2019;10(1):1914. doi:10.1038/s41467019-09839-x

44. Shen J, Abu-Amer Y, O'Keefe RJ, et al. Inflammation and epigenetic regulation in osteoarthritis. Connect Tissue Res. 2017;58(1):49-63. doi:10.1080/03008207.2016.1208655

45. McAllister MJ, Chemaly M, Eakin AJ, et al. NLRP3 as a potentially novel biomarker for the management of osteoarthritis. Osteoarthritis Cartilage. 2018;26(5):612-619. doi:10.1016/j.joca.2018.02.901
46. Rao Z, Chen X, Wu J, et al. Vitamin D receptor inhibits NLRP3 activation by impeding its BRCC3-mediated deubiquitination. Front Immunol. 2019;10:2783. doi:10.3389/fimmu.2019.02783

47. Wan P, Zhang Q, Liu W, et al. Cullin1 binds and promotes NLRP3 ubiquitination to repress systematic inflammasome activation. FASEB J. 2019;33(4):5793-5807. doi:10.1096/fj.201801681R

48. Loeser RF, Goldring SR, Scanzello CR, et al. Osteoarthritis: a disease of the joint as an organ. Arthritis Rheum. 2012;64(6):1697-1707. doi:10.1002/art.34453

49. Cao P, Li Y, Tang Y, et al. Pharmacotherapy for knee osteoarthritis: current and emerging therapies. Expert Opin Pharmacother. 2020;21 (7):797-809. doi:10.1080/14656566.2020.1732924

50. Hunter DJ, Jarcho JA. Viscosupplementation for osteoarthritis of the knee. $N$ Engl J Med. 2015;372(11):1040-1047. doi:10.1056/NEJMct 1215534

51. Deyle GD, Allen CS, Allison SC, et al. Physical therapy versus glucocorticoid injection for osteoarthritis of the knee. $N$ Engl $J$ Med. 2020;382(15):1420-1429. doi:10.1056/NEJMoa1905877

52. Zheng S, Wang B, Han W, et al. Vitamin D supplementation and inflammatory and metabolic biomarkers in patients with knee osteoarthritis: post hoc analysis of a randomised controlled trial. $\mathrm{Br}$ J Nutr. 2018;120(1):41-48. doi:10.1017/S0007114518001174

53. Gao XR, Chen YS, Deng W. The effect of vitamin D supplementation on knee osteoarthritis: a meta-analysis of randomized controlled trials. Int J Surg. 2017;46:14-20. doi:10.1016/j.ijsu.2017.08.010

54. Park CY. Vitamin D in the prevention and treatment of osteoarthritis: from clinical interventions to cellular evidence. Nutrients. 2019;11 (2):243. doi:10.3390/nu11020243

55. Li S, Niu G, Dong XN, et al. Vitamin D inhibits activities of metalloproteinase-9/-13 in articular cartilage in vivo and in vitro. J Nutr Sci Vitaminol. 2019;65(2):107-112. doi:10.3177/jnsv.65.107

56. Bonnet CS, Walsh DA. Osteoarthritis, angiogenesis and inflammation. Rheumatology. 2005;44(1):7-16. doi:10.1093/rheumatology/keh344

57. Kobayashi M, Squires GR, Mousa A, et al. Role of interleukin-1 and tumor necrosis factor alpha in matrix degradation of human osteoarthritic cartilage. Arthritis Rheum. 2005;52(1):128-135. doi:10.1002/art.20776

58. Youm YH, Nguyen KY, Grant RW, et al. The ketone metabolite $\beta$ hydroxybutyrate blocks NLRP3 inflammasome-mediated inflammatory disease. Nat Med. 2015;21(3):263-269. doi:10.1038/nm.3804

59. Jiang Y, Tuan RS. Origin and function of cartilage stem/progenitor cells in osteoarthritis. Nat Rev Rheumatol. 2015;11(4):206-212. doi:10.1038/nrrheum.2014.200
Journal of Inflammation Research

\section{Publish your work in this journal}

The Journal of Inflammation Research is an international, peerreviewed open-access journal that welcomes laboratory and clinical findings on the molecular basis, cell biology and pharmacology of inflammation including original research, reviews, symposium reports, hypothesis formation and commentaries on: acute/chronic inflammation; mediators of inflammation; cellular processes; molecular mechanisms; pharmacology and novel anti-inflammatory drugs; clinical conditions involving inflammation. The manuscript management system is completely online and includes a very quick and fair peerreview system. Visit http://www.dovepress.com/testimonials.php to read real quotes from published authors. 